\title{
MULTIGRID WAVEFORM RELAXATION FOR THE TIME-FRACTIONAL HEAT EQUATION*
}

\author{
FRANCISCO J. GASPAR ${ }^{\dagger}$ AND CARMEN RODRIGO
}

\begin{abstract}
In this work, we propose an efficient and robust multigrid method for solving the time-fractional heat equation. Due to the nonlocal property of fractional differential operators, numerical methods usually generate systems of equations for which the coefficient matrix is dense. Therefore, the design of efficient solvers for the numerical simulation of these problems is a difficult task. We develop a parallel-in-time multigrid algorithm based on the waveform relaxation approach, whose application to time-fractional problems seems very natural due to the fact that the fractional derivative at each spatial point depends on the values of the function at this point at all earlier times. Exploiting the Toeplitz-like structure of the coefficient matrix, the proposed multigrid waveform relaxation method has a computational cost of $O(N M \log (M))$ operations, where $M$ is the number of time steps and $N$ is the number of spatial grid points. A semialgebraic mode analysis is also developed to theoretically confirm the good results obtained. Several numerical experiments, including examples with nonsmooth solutions and a nonlinear problem with applications in porous media, are presented.
\end{abstract}

Key words. time-fractional heat equation, multigrid waveform relaxation, semialgebraic mode analysis

AMS subject classifications. 65F10, 65M22, 65M 55

DOI. $10.1137 / 16 \mathrm{M} 1090193$

1. Introduction. Fractional calculus has become increasingly popular in recent years due to its frequent appearance in various applications in fluid mechanics, signal processing, viscoelasticity, porous media flow, quantum mechanics, biology, medicine, physics, and engineering; see [8,15, 17, 20, 24, 32, 44], for example. In particular it has attracted much attention within the natural and social sciences, since it can properly model phenomena dominated by memory effects [29, 47] and problems exhibiting non-Markovian behavior in time.

A lot of effort has been focused on attempting to find robust and stable numerical and analytical methods for solving ordinary and partial differential equations of fractional order. The number of numerical analysis papers studying differential equations with fractional-order derivatives has risen dramatically in the past decade $[6,20,21,24,26,35,46]$. Due to the nonlocal property of the fractional differential operator, numerical methods usually generate systems of equations for which the coefficient matrix is dense. This is the main reason why most of these problems have been traditionally solved by Gaussian elimination, which requires a very high computational cost of $O\left(n^{3}\right)$ in addition to a high storage cost of $O\left(n^{2}\right)$, where $n$ is the total number of grid points. Some effort has been made to reduce this computational

*Submitted to the journal's Methods and Algorithms for Scientific Computing section August 18, 2016; accepted for publication (in revised form) April 13, 2017; published electronically July 12, 2017.

http://www.siam.org/journals/sisc/39-4/M109019.html

Funding: Francisco J. Gaspar has received funding from the European Union's Horizon 2020 research and innovation programme under the Marie Sklodowska-Curie grant agreement 705402, POROSOS. The work of Carmen Rodrigo is supported in part by the Spanish project FEDER /MCYT MTM2016-75139-R and the Diputación General de Aragón (Grupo consolidado PDIE).

${ }^{\dagger}$ CWI, Centrum Wiskunde and Informatica, 1098 XG Amsterdam, The Netherlands (gaspar@ cwi.nl, http://www.unizar.es/pde/fjgaspar/).

${ }_{\ddagger}^{\ddagger}$ IUMA and Applied Mathematics Department, University of Zaragoza, Zaragoza, Aragon 50009, Spain (carmenr@unizar.es). 
cost by approximating the coefficient matrix by a banded matrix [55], for example, obtaining a computational complexity of $O\left(n \log ^{2}(n)\right)$. This is quite different from integer differential operators, which typically yield sparse coefficient matrices that can be efficiently solved by fast iterative methods with $O(n)$ complexity. Therefore, the design of efficient solvers that reduce the computational cost is one of the challenges for the numerical simulation of fractional PDEs. For space-fractional PDEs, some efficient solvers, such as preconditioned Krylov subspace methods [40, 54] and multigrid methods [41], have already been proposed. The key is to take advantage of the Toeplitz-like structure of the coefficient matrix which arises from the discretization method proposed by Meerschaert and Tadjeran [31]. In this way, the storage requirements can be reduced to $O(N)$, and the matrix-vector multiplication can be done in $O(N \log (N))$ operations by using the fast Fourier transform (FFT). Recently, a fast solver based on a geometric multigrid method for nonuniform grids was proposed in [58]. The approach is based on the use of H-matrices to approximate the dense matrices. Regarding time-fractional PDEs, the coefficient matrix usually has an $M \times M$ block lower triangular Toeplitz structure, with each block of size $N \times N$, where $N$ is the number of spatial grid points and $M$ the number of time levels. A fast direct method taking advantage of the Toeplitz structure of the coefficient matrix is proposed in [22] with a complexity of $O\left(N M \log ^{2}(M)\right)$. As an alternative, in [36] the authors proposed the use of alternating direction implicit (ADI) schemes with a computational complexity of $O\left(N M^{2}\right)$. An approximate inversion method with $O(N M \log (M))$ was recently proposed in [23]. Their idea is to approximate the coefficient matrix by a block $\varepsilon$-circulant matrix, which can be block diagonalized by FFT. To solve the resulting complex block system, the authors use a multigrid method. Our main contribution is to propose an efficient and robust multigrid method based on the waveform relaxation approach to solve the time-fractional heat equation. Exploiting the Toeplitz-like structure of the coefficient matrix, the computational complexity of the proposed method is $O(N M \log (M))$, with a storage requirement of $O(N M)$, being only $O(M)$ for the storage of the coefficient matrix. Being the opposite of the method introduced in [23], the algorithm proposed here is directly applied to the original discretization of the problem, and also is better suited for nonlinear problems. We wish to emphasize that the proposed method is parallel in time, in contrast to the classical sequential time-integration methods based on time-stepping.

Waveform relaxation methods consist of continuous-in-time iterative algorithms for solving large systems of ordinary differential equations (ODEs). Their application to the solution of parabolic PDEs is based on the numerical method of lines, in which the spatial derivatives are replaced by discrete analogues obtaining a large system of ODEs, which is solved by standard iterative methods. The requirement of extra storage for unknowns represents a classical disadvantage of waveform methods; however, in our case this is not a drawback anymore since the time-fractional PDEs also need the solutions in previous time steps to be stored. Since the waveform relaxation method is based on the numerical method of lines, it is not clear how to combine it with techniques such as dynamic grid adaptation, although recently some effort has been carried out to combine parallel-in-time techniques with moving meshes [11, 16]. The convergence of the waveform relaxation methods was studied by Miekkala and Nevanlinna [33], who showed that the convergence could be too slow for the waveform relaxation to be competitive with standard time-stepping methods. Recently, some authors have investigated the convergence of some waveform relaxation methods for solving fractional differential equations [19]. We wish to point out that for timefractional PDEs, the fact that each spatial grid point at a fixed time is connected to 
all the values of the previous time steps makes the application of waveform relaxation methods to these problems very natural.

Multigrid methods (see $[45,48,56]$ for an introduction) are often used for the convergence acceleration of iterative methods, although they have a wider use and significance than just being acceleration techniques. These methods are among the most efficient methods for solving large algebraic systems arising from discretizations of PDEs, with optimal computational complexity, due to their ability to handle different scales present in the problem. Here, we propose the application of a multigrid approach based on the waveform relaxation method for solving time-fractional differential equations. This method combines the very fast multigrid convergence with the high parallel efficiency of waveform relaxation. Basically, it consists of applying a redblack zebra-in-time line relaxation together with a coarse-grid correction procedure based on coarsening only in the spatial dimension. Note that there is no coarsening in time in such a multigrid method, and the time is kept continuous. In this way, the coarsest grid is composed of only one spatial grid point and all the corresponding points in time. The multigrid waveform relaxation was first developed by Lubich and Ostermann [27], who showed that the basic waveform relaxation process can be accelerated by using the multigrid idea. Their work is based on the application of multigrid (in space) directly to the evolution equation. Since its introduction, this approach has been successfully applied to a variety of parabolic problems $[18,39,50,51,52,53]$, but never within a fractional context.

Local Fourier analysis (LFA), or local mode analysis [3, 5, 48, 56, 57], has become a very useful predictive tool for the analysis of the convergence of multigrid methods. The idea of this analysis is to focus on the local character of the operators involved in the multigrid algorithm, and to analyze their behavior on a basis of complex exponential functions. However, the failure of this analysis for the prediction of the multigrid convergence for convection-dominated or parabolic problems has been observed by different authors $[4,12,38]$. To overcome this difficulty, a semialgebraic mode analysis (SAMA) was proposed in [12]. This analysis, which is essentially a generalization of the classical local mode analysis, combines standard LFA with algebraic computation that accounts for the nonlocal character of the operators. It is clear that this is the approach that we should consider for the analysis of the multigrid waveform relaxation method for the time-fractional diffusion problem dealt with in this work. Notice the nonlocal character of this differential operator in time. Finally, we wish to emphasize that the proposed multigrid waveform relaxation method, as well as the SAMA for the study of its convergence, give rise to an efficient solution strategy for the time-fractional heat equation, which seems a very natural way to deal with this problem.

The remainder of this paper is organized as follows. Section 2 is devoted to introducing the considered one-dimensional model problem and its discretization. The proposed multigrid waveform relaxation method for its solution is described in section 3 . Next, the SAMA used for studying the convergence of this algorithm is explained in section 4 , together with some analysis results showing its suitability for the prediction of the behavior of the multigrid method. In section 5 the computational complexity of the proposed algorithm is discussed. After that, section 6 focuses on the generalization of the proposed methodology for a two-dimensional model problem. Finally, in section 7 , we illustrate the good behavior of the multigrid waveform relaxation method for solving the time-fractional diffusion problems considered in this work, by means of three different test problems, which include a nonlinear model problem with applications in porous media. Conclusions are drawn in section 8. 
2. Model problem and discretization. We consider the time-fractional heat equation, arising by replacing the first-order time derivative with the Caputo derivative of order $\delta$, where $0<\delta<1$. In this section, we restrict ourselves to the one-dimensional case for simplicity in the presentation. Therefore, we can formulate our model problem as the following initial-boundary value problem:

$$
\begin{array}{r}
D_{t}^{\delta} u-\frac{\partial^{2} u}{\partial x^{2}}=f(x, t), \quad 0<x<L, t>0 \\
u(0, t)=0, u(L, t)=0, \quad t>0 \\
u(x, 0)=g(x), \quad 0 \leq x \leq L .
\end{array}
$$

As mentioned above, $D_{t}^{\delta}$ denotes the Caputo fractional derivative, defined as follows $[10,46]$ :

$$
D_{t}^{\delta} u(x, t):=\left[J^{1-\delta}\left(\frac{\partial u}{\partial t}\right)\right](x, t), \quad 0 \leq x \leq L, t>0,
$$

where $J^{1-\delta}$ represents the Riemann-Liouville fractional integral operator of order $1-\delta$, given by

$$
\left(J^{1-\delta} u\right)(x, t):=\left[\frac{1}{\Gamma(1-\delta)} \int_{0}^{t}(t-s)^{-\delta} u(x, s) d s\right], \quad 0 \leq x \leq L, t>0,
$$

where $\Gamma$ is the Gamma function [9].

Model problem (1)-(3) is discretized on a uniform rectangular mesh $G_{h, \tau}=G_{h} \times$ $G_{\tau}$, with

$$
\begin{aligned}
& G_{h}=\left\{x_{n}=n h, n=0,1, \ldots, N+1\right\}, \\
& G_{\tau}=\left\{t_{m}=m \tau, m=0,1, \ldots, M\right\},
\end{aligned}
$$

where $h=\frac{L}{N+1}, \tau=\frac{T}{M}$ with $T$ the final time, and $N+1$ and $M$ are positive integers representing the number of subdivisions in the spatial and temporal intervals, respectively. We denote by $u_{n, m}$ the nodal approximation to the solution at each grid point $\left(x_{n}, t_{m}\right)$.

In the nodal points, the Caputo fractional derivative $D_{t}^{\delta} u$ can be written as

$$
D_{t}^{\delta} u\left(x_{n}, t_{m}\right)=\frac{1}{\Gamma(1-\delta)} \sum_{k=0}^{m-1} \int_{t_{k}}^{t_{k+1}}\left(t_{m}-s\right)^{-\delta} \frac{\partial u\left(x_{n}, s\right)}{\partial t} d s
$$

and it is approximated by using the well-known L1 scheme [37], which uses $\frac{\partial u\left(x_{n}, s\right)}{\partial t} \approx$ $\frac{u_{n, k+1}-u_{n, k}}{\tau}, t_{k} \leq s \leq t_{k+1}$, to obtain

$$
\begin{aligned}
D_{M}^{\delta} u_{n, m} & :=\frac{1}{\Gamma(1-\delta)} \sum_{k=0}^{m-1} \frac{u_{n, k+1}-u_{n, k}}{\tau} \int_{t_{k}}^{t_{k+1}}\left(t_{m}-s\right)^{-\delta} d s \\
& =\frac{\tau^{-\delta}}{\Gamma(2-\delta)}\left[d_{1} u_{n, m}-d_{m} u_{n, 0}+\sum_{k=1}^{m-1}\left(d_{k+1}-d_{k}\right) u_{n, m-k}\right]
\end{aligned}
$$

by defining $d_{k}=k^{1-\delta}-(k-1)^{1-\delta}, k \geq 1$. 
Regarding the diffusive term, we use standard central finite differences to approximate the spatial derivatives. Summarizing, we deal with the following discrete problem:

$$
\begin{aligned}
D_{M}^{\delta} u_{n, m}-\frac{u_{n+1, m}-2 u_{n, m}+u_{n-1, m}}{h^{2}} & =f\left(x_{n}, t_{m}\right), \quad 1 \leq n \leq N, 1 \leq m \leq M, \\
u_{0, m} & =0, \quad u_{N+1, m}=0,0<m \leq M, \\
u_{n, 0} & =g\left(x_{n}\right), \quad 0 \leq n \leq N+1 .
\end{aligned}
$$

3. Multigrid waveform relaxation in one dimension. For solving timedependent PDEs, the multigrid waveform relaxation method uses the numerical method of lines, replacing any spatial derivative by discrete formulas (obtained by the finite element, finite difference, or finite volume methods) in the discrete spatial domain. Thus, the PDE is transformed into a large set of ODEs. In our case, that is, considering time-fractional derivatives of order $\delta$, we have

$$
D_{t}^{\delta} u_{h}(t)+A_{h} u_{h}(t)=f_{h}(t), u_{h}(0)=g_{h}, t>0,
$$

where $u_{h}$ and $f_{h}$ are functions of time $t$ defined on a discrete spatial mesh, and $A_{h}$ is the discrete approximation in space of the continuous operator defining the considered PDE. Since discrete operators are usually represented by matrices and grid functions by vectors, in the following we will use either the terminology of discrete differential operators and grid functions or that of matrices and vectors. The next step is the solution of the large system of ODEs by an iterative algorithm. For instance, if we consider a splitting of the spatial discrete operator $A_{h}=M_{h}-N_{h}$, one step of the iterative scheme for (14) can be written as

$$
D_{t}^{\delta} u_{h}^{k}(t)+M_{h} u_{h}^{k}(t)=N_{h} u_{h}^{k-1}(t)+f_{h}(t), u_{h}^{k}(0)=g_{h} \text { for } k \geq 1,
$$

where $u_{h}^{k}(t)$ denotes the approximation obtained at iteration $k$. The initial iterate $u_{h}^{0}(t)$ is defined along the whole time interval, it being natural to choose a constant initial approximation equal to the initial condition in (14), that is, $u_{h}^{0}(t)=g_{h}, t>0$.

In this work, for the one-dimensional problem, we will consider a red-black GaussSeidel iteration which consists of a two-stage procedure, given by

$$
\begin{aligned}
& D_{t}^{\delta} u_{n}^{k}(t)+\frac{2}{h^{2}} u_{n}^{k}(t)=\frac{1}{h^{2}}\left(u_{n-1}^{k-1}(t)+u_{n+1}^{k-1}(t)\right)+f_{n}(t) \quad \text { if } n \text { is even, } \\
& D_{t}^{\delta} u_{n}^{k}(t)-\frac{1}{h^{2}}\left(u_{n-1}^{k}(t)-2 u_{n}^{k}(t)+u_{n+1}^{k}(t)\right)=f_{n}(t) \quad \text { if } n \text { is odd }
\end{aligned}
$$

that is, first the even points in space are visited, and after that we solve the unknowns in the grid points with odd numbering.

To accelerate the convergence of the red-black Gauss-Seidel waveform relaxation, a coarse-grid correction process based on a coarsening procedure only in the spatial dimension is performed, resulting in the so-called linear multigrid waveform relaxation algorithm [49]. This method consists essentially of the standard multigrid algorithm but applied to systems of ODEs, such as the one in (14). Considering the standard full-weighting restriction and the linear interpolation as transfer-grid operators, the algorithm of the multigrid waveform relaxation (WRMG) is given in Algorithm 1.

After discretizing in time, that is, replacing the differential operator $D_{t}^{\delta}$ by $D_{M}^{\delta}$, the previous algorithm can be interpreted as a space-time multigrid method with coarsening only in space. Thus, the red-black Gauss-Seidel waveform relaxation can 


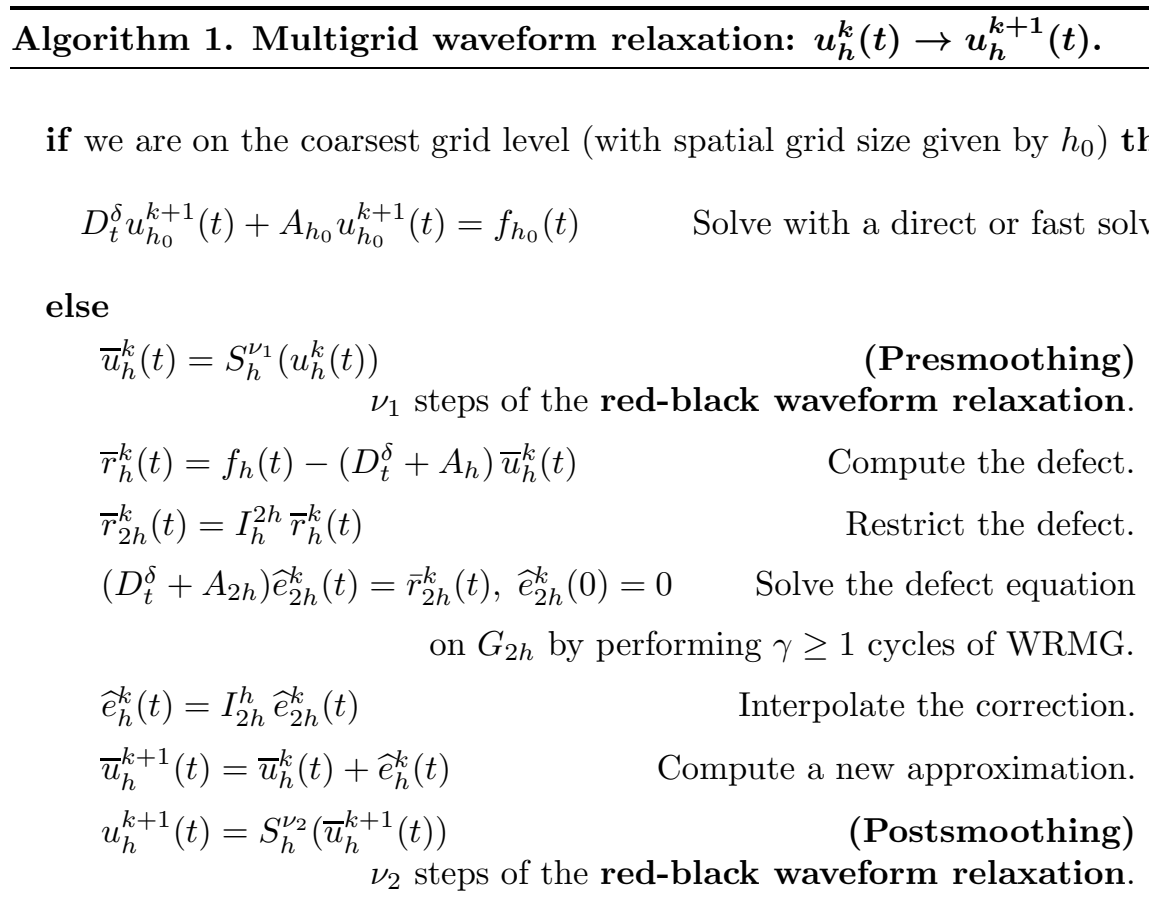

end if

be seen as a zebra-in-time line relaxation, and standard full-weighting restriction and linear interpolation in space are considered for the data transfer between the levels in the multigrid hierarchy. Thus, the whole multigrid waveform relaxation combines a zebra-in-time line relaxation with a standard semicoarsening strategy only in the spatial dimension.

4. Semialgebraic mode analysis in one dimension. The analysis that we perform here is based on an exponential Fourier mode analysis or local Fourier analysis (LFA) technique only in space and an exact analytical approach in time. This kind of semialgebraic mode analysis (SAMA) was introduced for the first time in [12], where the authors mainly study the convergence of multigrid methods on space-time grids for parabolic problems. Furthermore, they extend the application of this analysis to nonparabolic problems like elliptic diffusion in layered media and convection diffusion. The main idea of this analysis is to study the evolution of the spatial Fourier modes over time. This semialgebraic analysis provides very accurate predictions of the performance of multigrid methods, and indeed it can be made rigorous if appropriate boundary conditions are considered. Next, we describe the basics of this analysis. Although in [12] the authors give a detailed explanation, here we present a slightly different description of this analysis.

4.1. Basics of the analysis. It is well known that LFA assumes the formal extension to all multigrid components to an infinite grid, neglecting the boundary conditions, and considers discrete linear operators with constant coefficients. Therefore, we define the following infinite grid:

$$
\mathcal{G}_{h}=\left\{x_{n}=n h, n \in \mathbb{Z}\right\},
$$


where $h$ is the spatial discretization step. For a fixed $t$, any discrete grid function $u_{h}(\cdot, t)$ defined on $\mathcal{G}_{h}$ can be written as a formal linear combination of the so-called Fourier modes given by $\varphi_{h}(\theta, x)=e^{\imath \theta x}$, where $\theta \in \Theta_{h}=(-\pi / h, \pi / h]$, that is,

$$
u_{h}(x, t)=\sum_{\theta \in \Theta_{h}} c_{\theta}(t) \varphi_{h}(\theta, x), \quad x \in \mathcal{G}_{h} .
$$

Notice that coefficients $c_{\theta}(t)$ depend on the time variable. The Fourier modes, which generate the so-called Fourier space $\mathcal{F}\left(\mathcal{G}_{h}\right)=\left\{\varphi_{h}(\theta, \cdot), \theta \in \Theta_{h}\right\}$, become eigenfunctions of any discrete operator with constant coefficients defined formally on $\mathcal{G}_{h}$. For

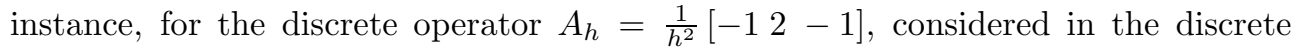
model problem (11), it is fulfilled that

$$
A_{h} \varphi_{h}(\theta, \cdot)=\widehat{A}_{h}(\theta) \varphi_{h}(\theta, \cdot)
$$

where

$$
\widehat{A}_{h}(\theta)=\frac{4}{h^{2}} \sin \left(\frac{\theta h}{2}\right)
$$

is the Fourier representation of operator $A_{h}$, which is also called the Fourier symbol of $A_{h}$.

The aim of the local Fourier analysis is to analyze how the operators involved in the multigrid algorithm act on such Fourier modes. We can study how efficiently the relaxation process eliminates the high-frequency components of the error through a smoothing analysis, or how the two-grid operator acts on the Fourier space through a two-grid analysis.

First, we proceed to explain the smoothing analysis for a standard relaxation procedure. After that, we describe the analysis for the coarse-grid correction operator, and finally we combine both analyses in order to perform a complete study of the two-grid cycle. For this purpose, we need to distinguish between high- and lowfrequency components. This classification is done with respect to the coarsening strategy, which is chosen as standard coarsening, that is, the step size is double on the coarse grid, which is denoted by $\mathcal{G}_{2 h}$. Recall that in a typical multigrid waveform relaxation procedure the coarsening applies only in the spatial domain. The space of low frequencies is defined as $\Theta_{2 h}=(-\pi / 2 h, \pi / 2 h]$, and the high frequencies are given by $\Theta_{h} \backslash \Theta_{2 h}$.

Smoothing analysis. We describe the semialgebraic smoothing analysis for a standard relaxation procedure based on a decomposition of the spatial discrete operator $A_{h}$ as $A_{h}=M_{h}-N_{h}$. Denoting by $e_{h}^{k}(\cdot, t)$ and $e_{h}^{k-1}(\cdot, t)$ the error grid functions at the $k$ and $k-1$ iterations of this procedure, an iteration of this waveform relaxation method is given by

$$
D_{t}^{\delta} e_{h}^{k}(x, t)+M_{h} e_{h}^{k}(x, t)=N_{h} e_{h}^{k-1}(x, t) \text { for } k \geq 1 \text {, and } x \in \mathcal{G}_{h}, t>0,
$$

with initial condition $e_{h}^{k}(x, 0)=0, x \in \mathcal{G}_{h}$.

From (19), we can write the error at the $j$ iteration, $e_{h}^{j}(x, t)$, in the following way,

$$
e_{h}^{j}(x, t)=\sum_{\theta \in \Theta_{h}} c_{\theta}^{j}(t) \varphi_{h}(\theta, x), \quad x \in \mathcal{G}_{h}, t>0,
$$

and then by using that $\varphi_{h}(\theta, x)$ are eigenfunctions of operators $M_{h}$ and $N_{h}$ (that is, $M_{h} \varphi_{h}(\theta, x)=\widehat{M}_{h}(\theta) \varphi_{h}(\theta, x)$, for example, where $\widehat{M}_{h}(\theta)$ is the symbol of $\left.M_{h}\right)$, it 
follows for each frequency $\theta \in \Theta_{h}$ that

$$
D_{t}^{\delta} c_{\theta}^{k}(t)+\widehat{M}_{h}(\theta) c_{\theta}^{k}(t)=\widehat{N}_{h}(\theta) c_{\theta}^{k-1}(t) \text { for } k \geq 1, t>0 .
$$

Considering the discretization of $D_{t}^{\delta}$ on the uniform grid $G_{\tau}, D_{M}^{\delta}$, defined in (10), and denoting by $\left(c_{\theta}^{k, 1}, \ldots, c_{\theta}^{k, M}\right)$ the approximation of $c_{\theta}^{k}(t)$ on grid $G_{\tau}$, we obtain the relation

$$
\left(\begin{array}{c}
c_{\theta}^{k, 1} \\
c_{\theta}^{k, 2} \\
\vdots \\
c_{\theta}^{k, M}
\end{array}\right)=\widetilde{\mathcal{M}}_{h, \tau}^{-1}(\theta) \widetilde{\mathcal{N}}_{h, \tau}(\theta)\left(\begin{array}{c}
c_{\theta}^{k-1,1} \\
c_{\theta}^{k-1,2} \\
\vdots \\
c_{\theta}^{k-1, M}
\end{array}\right)
$$

where $\widetilde{\mathcal{N}}_{h, \tau}(\theta)=\operatorname{diag}\left(\widehat{N}_{h}(\theta)\right)$, and

$$
\widetilde{\mathcal{M}}_{h, \tau}(\theta)=\left(\begin{array}{cccc}
r_{1}+\widehat{M}_{h}(\theta) & 0 & \cdots & 0 \\
r_{2} & r_{1}+\widehat{M}_{h}(\theta) & \cdots & 0 \\
\vdots & \ddots & \ddots & \vdots \\
r_{M} & \cdots & r_{2} & r_{1}+\widehat{M}_{h}(\theta)
\end{array}\right),
$$

with $r_{i}=\frac{\tau^{-\delta}}{\Gamma(2-\delta)}\left(d_{i}-d_{i-1}\right), i=1, \ldots, M$, assuming $d_{0}=0$.

Denoting $\widetilde{\mathcal{S}}_{h, \tau}(\theta)=\widetilde{\mathcal{M}}_{h, \tau}^{-1}(\theta) \widetilde{\mathcal{N}}_{h, \tau}(\theta)$, we can define the smoothing factor of the relaxation procedure as follows:

$$
\mu=\sup _{\Theta_{h} \backslash \Theta_{2 h}}\left(\rho\left(\widetilde{\mathcal{S}}_{h, \tau}(\theta)\right)\right) .
$$

Coarse-grid correction analysis. We now proceed to explain the analysis of the coarse-grid correction method. An error $e_{h}^{k}$ is transformed by this method as $e_{h}^{k+1}=C_{h}^{2 h} e_{h}^{k}$, where $C_{h}^{2 h}=I_{h}-I_{2 h}^{h}\left(D_{t}^{\delta}+A_{2 h}\right)^{-1} I_{h}^{2 h}\left(D_{t}^{\delta}+A_{h}\right)$ is the coarse-grid correction operator. Here $I_{h}$ is the identity operator, $D_{t}^{\delta}+A_{h}$ and $D_{t}^{\delta}+A_{2 h}$ are the fine- and coarse-grid operators, respectively, and $I_{2 h}^{h}$ and $I_{h}^{2 h}$ are transfer operators from coarse to fine grids, and vice versa.

As we have chosen standard coarsening, the fine-grid Fourier mode $\varphi_{h}(\theta, x)$ when injected into the coarse grid aliases with the coarse-grid Fourier mode $\varphi_{2 h}(2 \theta, x)$. Thus, for any low frequency $\theta^{0} \in \Theta_{2 h}$ we define the high frequency $\theta^{1}=\theta^{0}-$ $\operatorname{sign}\left(\theta^{0}\right) \pi / h$. Taking this into account, the Fourier space is decomposed into twodimensional subspaces, known as $2 h$-harmonics (see $[48,57]$ for more details):

$$
\mathcal{F}^{2}(\theta)=\operatorname{span}\left\{\varphi_{h}\left(\theta^{0}, \cdot\right), \varphi_{h}\left(\theta^{1}, \cdot\right)\right\}, \quad \theta=\theta^{0} \in \Theta_{2 h} .
$$

The coarse-grid correction operator $C_{h}^{2 h}$ leaves the two-dimensional subspace of harmonics $\mathcal{F}^{2}\left(\theta^{0}\right)$ invariant for an arbitrary Fourier frequency $\theta^{0} \in \Theta_{2 h}$. Let us define for any $\theta^{0} \in \Theta_{2 h}$ the vector $\varphi_{h}\left(\theta^{0}, \cdot\right)=\left(\varphi_{h}\left(\theta^{0}, \cdot\right), \varphi_{h}\left(\theta^{1}, \cdot\right)\right)$. As the error at the iteration $k$ can be written as $e_{h}^{k}(x, t)=\sum_{\theta \in \Theta_{2 h}} \mathbf{c}_{\theta}^{k}(t) \boldsymbol{\varphi}_{h}(\theta, x)^{T}$, with $\mathbf{c}_{\theta}^{k}(t)=\left(c_{\theta^{0}}^{k}(t), c_{\theta^{1}}^{k}(t)\right)$, the error at the iteration $k+1$ after application of the coarse-grid correction method is given by $\sum_{\theta \in \Theta_{2 h}} \widehat{C}_{h}^{2 h}(\theta) \mathbf{c}_{\theta}^{k}(t) \varphi_{h}(\theta, \cdot)^{T}$, where $\widehat{C}_{h}^{2 h}(\theta)$ is a $2 \times 2$ matrix given by the expression

$$
\widehat{C}_{h}^{2 h}(\theta)=I_{2}-\widehat{I}_{2 h}^{h}(\theta)\left(D_{t}^{\delta}+\widehat{A}_{2 h}(\theta)\right)^{-1} \widehat{I}_{h}^{2 h}(\theta)\left(D_{t}^{\delta}+\widehat{A}_{h}(\theta)\right)
$$


where $I_{2}$ is the $2 \times 2$ identity matrix, and $\widehat{A}_{h}(\theta), \widehat{A}_{2 h}(\theta), \widehat{I}_{2 h}^{h}(\theta), \widehat{I}_{h}^{2 h}(\theta)$ denote the symbols of the fine- and coarse-grid spatial operators, the prolongation operator, and the restriction operator, respectively. The Fourier symbol of the fine-grid operator is given by $\widehat{A}_{h}(\theta)=\operatorname{diag}\left(\widehat{A}_{h}\left(\theta^{0}\right), \widehat{A}_{h}\left(\theta^{1}\right)\right)$, and the symbol of the coarse-grid operator by $\widehat{A}_{2 h}(\theta)$. The Fourier symbols of the prolongation and restriction operators for $\theta=\theta^{0} \in \Theta_{2 h}$ are given by

$$
\widehat{I}_{2 h}^{h}(\theta)=\left(\begin{array}{c}
\widehat{I}_{2 h}^{h}\left(\theta^{0}\right) \\
\widehat{I}_{2 h}^{h}\left(\theta^{1}\right)
\end{array}\right), \quad \widehat{I}_{h}^{2 h}(\theta)=\left(\widehat{I}_{h}^{2 h}\left(\theta^{0}\right), \widehat{I}_{h}^{2 h}\left(\theta^{1}\right)\right) .
$$

Let us suppose that the error at the iteration $k$ is given by

$$
\mathbf{c}_{\theta}^{k}(t) \boldsymbol{\varphi}_{h}(\theta, \cdot)^{T}=c_{\theta^{0}}^{k}(t) \varphi_{h}\left(\theta^{0}, \cdot\right)+c_{\theta^{1}}^{k}(t) \varphi_{h}\left(\theta^{1}, \cdot\right) .
$$

By considering the discretization of $D_{t}^{\delta}$ on the uniform grid $G_{\tau}, D_{M}^{\delta}$, defined in (10), we obtain that the error after application of the coarse-grid correction is given by $\widetilde{\mathcal{C}}_{h, \tau}^{2 h}(\theta) \mathbf{c}_{\theta}^{k}(t) \boldsymbol{\varphi}_{h}(\theta, \cdot)^{T}$, with $\widetilde{\mathcal{C}}_{h, \tau}^{2 h}(\theta)$ a $2 M \times 2 M$ matrix, given by

$$
\widetilde{\mathcal{C}}_{h, \tau}^{2 h}(\theta)=I_{2 M}-\widetilde{\mathcal{I}}_{2 h}^{h}(\theta)\left(\widetilde{\mathcal{A}}_{2 h, \tau}(\theta)\right)^{-1} \widetilde{\mathcal{I}}_{h}^{2 h}(\theta) \widetilde{\mathcal{A}}_{h, \tau}(\theta) .
$$

Here, $I_{2 M}$ is the identity matrix of order $2 M, \widetilde{\mathcal{A}}_{h, \tau}(\theta)$ is the $2 M \times 2 M$ matrix

$$
\widetilde{\mathcal{A}}_{h, \tau}(\theta)=\left(\begin{array}{cc}
\widetilde{\mathcal{A}}_{h, \tau}\left(\theta^{0}\right) & 0 \\
0 & \widetilde{\mathcal{A}}_{h, \tau}\left(\theta^{1}\right)
\end{array}\right), \quad \theta=\theta^{0} \in \Theta_{2 h},
$$

where for $\alpha=0,1$,

$$
\widetilde{\mathcal{A}}_{h, \tau}\left(\theta^{\alpha}\right)=\left(\begin{array}{cccc}
r_{1}+\widehat{A}_{h}\left(\theta^{\alpha}\right) & 0 & \cdots & 0 \\
r_{2} & r_{1}+\widehat{A}_{h}\left(\theta^{\alpha}\right) & \cdots & 0 \\
\vdots & \ddots & \ddots & \vdots \\
r_{M} & \cdots & r_{2} & r_{1}+\widehat{\widehat{A}}_{h}\left(\theta^{\alpha}\right)
\end{array}\right),
$$

with $\widehat{A}_{h}\left(\theta^{\alpha}\right)$ the symbol of the fine-grid spatial operator, and $r_{i}=\frac{\tau^{-\delta}}{\Gamma(2-\delta)}\left(d_{i}-d_{i-1}\right)$ for $i=1, \ldots, M$, assuming $d_{0}=0$.

About the restriction and interpolation, $\widetilde{\mathcal{I}}_{h}^{2 h}(\theta)$ is the $M \times 2 M$ matrix

$$
\widetilde{\mathcal{I}}_{h}^{2 h}(\theta)=\left[\widehat{I}_{h}^{2 h}\left(\theta^{0}\right) I_{M}, \widehat{I}_{h}^{2 h}\left(\theta^{1}\right) I_{M}\right],
$$

and $\widetilde{\mathcal{I}}_{2 h}^{h}(\theta)$ is the $2 M \times M$ matrix

$$
\widetilde{\mathcal{I}}_{2 h}^{h}(\theta)=\left[\widehat{I}_{2 h}^{h}\left(\theta^{0}\right) I_{M}, \widehat{I}_{2 h}^{h}\left(\theta^{1}\right) I_{M}\right]^{T} .
$$

Two-grid analysis. Combining the Fourier smoothing analysis and the Fourier coarse-grid correction analysis previously introduced, we perform the semialgebraic two-grid analysis. The two-grid operator $\mathcal{T}_{h, \tau}^{2 h}$ is defined as $\mathcal{T}_{h, \tau}^{2 h}=\mathcal{S}_{h, \tau}^{\nu_{2}} \mathcal{C}_{h, \tau}^{2 h} \mathcal{S}_{h, \tau}^{\nu_{1}}$, where $\mathcal{C}_{h, \tau}^{2 h}$ is the coarse-grid operator, $\mathcal{S}_{h, \tau}$ is a smoothing operator, and $\nu_{1}, \nu_{2}$ indicate the number of pre- and postsmoothing steps, respectively.

We recall that the coarse-grid correction operator $\mathcal{C}_{h, \tau}^{2 h}$ leaves the two-dimensional subspaces of harmonics $\mathcal{F}^{2}(\theta)$ invariant for an arbitrary Fourier frequency $\theta=\theta^{0} \in$ 
$\Theta_{2 h}$. This same invariance property is true for the smoothers $\mathcal{S}_{h, \tau}$ considered in this work. Therefore, the two-grid operator $\mathcal{T}_{h, \tau}^{2 h}$ also leaves the $2 h$-harmonic subspaces invariant.

Let us suppose that the error at iteration $k$ is given by $\mathbf{c}_{\theta}^{k}(t) \varphi_{h}(\theta, \cdot)^{T}=$ $c_{\theta^{0}}^{k}(t) \varphi_{h}\left(\theta^{0}, \cdot\right)+c_{\theta^{1}}^{k}(t) \varphi_{h}\left(\theta^{1}, \cdot\right)$. By considering the discretization of $D_{t}^{\delta}$ on the uniform grid $G_{\tau}, D_{M}^{\delta}$, defined in (10), we obtain that the error after application of the two-grid method is given by $\widetilde{\mathcal{T}}_{h, \tau}^{2 h}(\theta) \mathbf{c}_{\theta}^{k}(t) \boldsymbol{\varphi}_{h}(\theta, \cdot)^{T}$, with $\widetilde{\mathcal{T}}_{h, \tau}^{2 h}(\theta)$ a $2 M \times 2 M$ matrix, given by

$$
\widetilde{\mathcal{T}}_{h, \tau}^{2 h}(\theta)=\widetilde{\mathcal{S}}_{h, \tau}^{\nu_{2}}(\theta)\left(I_{2 M}-\widetilde{\mathcal{I}}_{2 h}^{h}(\theta)\left(\widetilde{\mathcal{A}}_{2 h, \tau}(\theta)\right)^{-1} \widetilde{\mathcal{I}}_{h}^{2 h}(\theta) \widetilde{\mathcal{A}}_{h, \tau}(\theta)\right) \widetilde{\mathcal{S}}_{h, \tau}^{\nu_{1}}(\theta) .
$$

If the chosen smoother is an iterative method which does not couple frequencies, then $\widetilde{\mathcal{S}}_{h, \tau}(\theta)$ is the $2 M \times 2 M$ matrix

$$
\widetilde{\mathcal{S}}_{h, \tau}(\theta)=\left(\begin{array}{cc}
\widetilde{\mathcal{S}}_{h, \tau}\left(\theta^{0}\right) & 0 \\
0 & \widetilde{\mathcal{S}}_{h, \tau}\left(\theta^{1}\right)
\end{array}\right),
$$

where, for $\alpha=0,1, \widetilde{\mathcal{S}}_{h, \tau}\left(\theta^{\alpha}\right)$ is given as previously.

In the case of a pattern waveform relaxation method, such as the red-black waveform relaxation, it is well known that the smoother couples frequencies but leaves invariant the two-dimensional subspaces $\mathcal{F}^{2}(\theta)$. In particular, for the redblack waveform relaxation considered in this work, the symbol is given by $\widetilde{\mathcal{S}}_{h, \tau}(\theta)=$ $\widetilde{\mathcal{S}}_{h, \tau}^{\text {black }}(\theta) \widetilde{\mathcal{S}}_{h, \tau}^{\text {red }}(\theta)$, where $\widetilde{\mathcal{S}}_{h, \tau}^{\text {black }}(\theta)$ and $\widetilde{\mathcal{S}}_{h, \tau}^{\text {red }}(\theta)$ are $2 M \times 2 M$ matrices coupling frequencies $\theta^{0}$ and $\theta^{1}$. More concretely,

$$
\begin{gathered}
\widetilde{\mathcal{S}}_{h, \tau}^{\text {red }}(\theta)=\frac{1}{2}\left(\begin{array}{cc}
\widetilde{\mathcal{M}}_{h, \tau}^{-1}\left(\theta^{0}\right) \widetilde{\mathcal{N}}_{h, \tau}\left(\theta^{0}\right)+I_{M} & \widetilde{\mathcal{M}}_{h, \tau}^{-1}\left(\theta^{1}\right) \widetilde{\mathcal{N}}_{h, \tau}\left(\theta^{1}\right)-I_{M} \\
\widetilde{\mathcal{M}}_{h, \tau}^{-1}\left(\theta^{0}\right) \widetilde{\mathcal{N}}_{h, \tau}\left(\theta^{0}\right)-I_{M} & \widetilde{\mathcal{M}}_{h, \tau}^{-1}\left(\theta^{1}\right) \widetilde{\mathcal{N}}_{h, \tau}\left(\theta^{1}\right)+I_{M}
\end{array}\right), \\
\widetilde{\mathcal{S}}_{h, \tau}^{\text {black }}(\theta)=\frac{1}{2}\left(\begin{array}{cc}
\widetilde{\mathcal{M}}_{h, \tau}^{-1}\left(\theta^{0}\right) \widetilde{\mathcal{N}}_{h, \tau}\left(\theta^{0}\right)+I_{M} & -\widetilde{\mathcal{M}}_{h, \tau}^{-1}\left(\theta^{1}\right) \widetilde{\mathcal{N}}_{h, \tau}\left(\theta^{1}\right)+I_{M} \\
-\widetilde{\mathcal{M}}_{h, \tau}^{-1}\left(\theta^{0}\right) \widetilde{\mathcal{N}}_{h, \tau}\left(\theta^{0}\right)+I_{M} & \widetilde{\mathcal{M}}_{h, \tau}^{-1}\left(\theta^{1}\right) \widetilde{\mathcal{N}}_{h, \tau}\left(\theta^{1}\right)+I_{M}
\end{array}\right),
\end{gathered}
$$

where $I_{M}$ is the identity matrix of size $M \times M$, and $\widetilde{\mathcal{M}}_{h, \tau}(\theta)$ and $\widetilde{\mathcal{N}}_{h, \tau}(\theta)$ are given as explained in the smoothing analysis section, using that $M_{h}$ is the diagonal part of matrix $A_{h}$, typical for a Jacobi-type relaxation. For a more detailed explanation of the SAMA for this smoother, we refer the reader to [12].

Finally, the convergence factor of the two-grid method can be estimated as

$$
\rho=\sup _{\Theta_{2 h}}\left(\rho\left(\widetilde{\mathcal{T}}_{h, \tau}^{2 h}(\theta)\right)\right),
$$

4.2. Analysis results. This section is focused on the analysis of the robustness of the proposed multigrid waveform relaxation method for the considered problem. When studying the multigrid convergence for the standard heat equation, it is well known that parameter $\tau / h^{2}$ describes the anisotropy in the operator, resulting in the relevant parameter for its analysis; see, e.g., [13]. However, as it can be observed in Figure 1, this parameter is not the important one for the time-fractional heat equation. In Figure 1, we depict the two-grid convergence factors provided by the SAMA for a range of values of parameter $\tau / h^{2}$ from $2^{-12}$ to $2^{12}$, for different fractional orders $\delta$. Only one smoothing step is considered, and the zebra-in-time smoother is used as previously described. It is clearly seen that, although the convergence rates are 


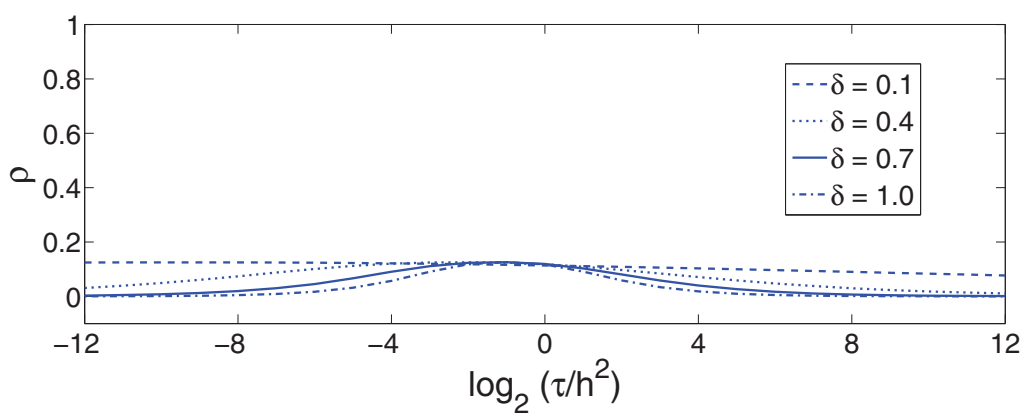

FIG. 1. Two-grid convergence factors predicted by the analysis for different values of parameter $\lambda=\tau / h^{2}$ from $2^{-12}$ to $2^{12}$ and different fractional orders $\delta$.

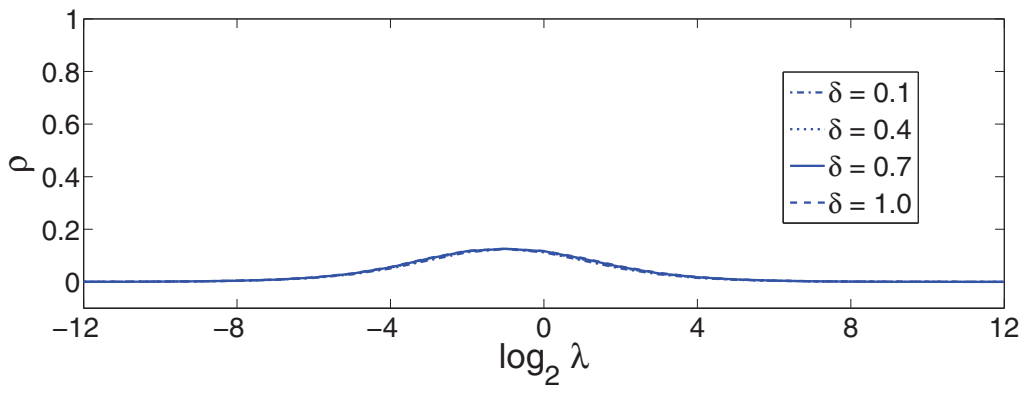

FIG. 2. Two-grid convergence factors predicted by the analysis for different values of parameter $\lambda=\tau^{\delta} \Gamma(2-\delta) / h^{2}$ and various fractional orders $\delta$.

bounded by 0.2 for all cases, we do not obtain a $\delta$-independent convergence for a fixed value of $\tau / h^{2}$. In this case, the relevant parameter is $\lambda=\tau^{\delta} \Gamma(2-\delta) / h^{2}$, as shown in Figure 2, where it is observed that the obtained multigrid convergence becomes robust for any value of $\delta$ with respect to parameter $\lambda$. In this figure, the number of time steps is chosen as $M=32$. Notice that, for any fixed value of $\delta$, the multigrid convergence is satisfactory for any value of parameter $\lambda$, which is very important for the global behavior of the method since this parameter will vary from grid level to grid level within the multigrid algorithm. The corresponding MATLAB function used to carry out the SAMA results in this figure is available in the supplementary material.

The results obtained by the SAMA match very accurately the real asymptotic convergence factors experimentally computed. This can be seen in Figure 3, where the two-grid convergence factors predicted by the analysis (denoted as $\rho$ and displayed as a solid line) are compared with those asymptotic convergence factors experimentally computed (represented by $\rho_{h}$ and depicted by circles). To compute the latter, we consider a grid of size $256 \times 32$, and we use a $W$-cycle, a random initial guess, and a zero right-hand side in order to avoid round-off errors. We can see in the picture a very accurate prediction of the SAMA, making it very useful for the analysis of the proposed WRMG method.

Finally, we would like to show that the behavior of the proposed multigrid waveform relaxation is very satisfactory with respect to the number of time levels considered. Since it is usually sufficient to analyze the behavior of the two-grid method to estimate the convergence of the multigrid method (see [48]), in Table 1 we show the two-grid convergence factors provided by the analysis by considering a wide range of 


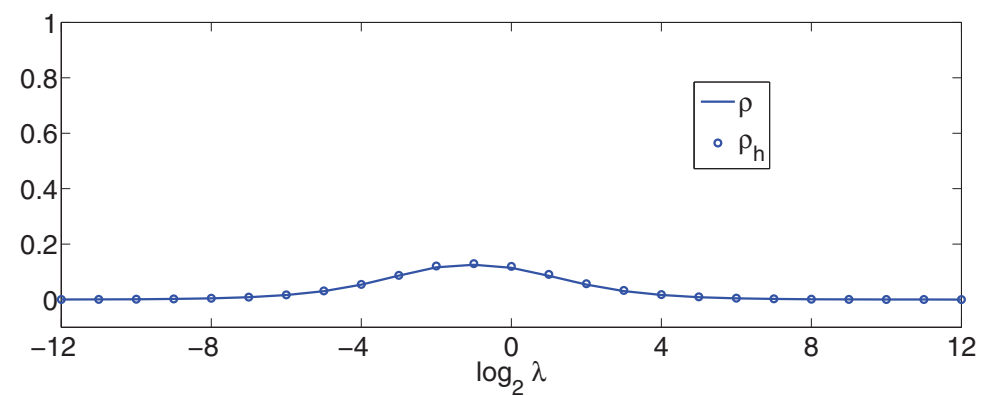

FIG. 3. Comparison between the two-grid convergence factors predicted by the analysis $(\rho)$ and the asymptotic convergence factor of a $W$-cycle experimentally computed $\left(\rho_{h}\right)$ for different values of parameter $\lambda=\tau^{\delta} \Gamma(2-\delta) / h^{2}$ and fractional order $\delta=0.4$.

TABLE 1

Two-grid convergence factors predicted by the analysis together with the corresponding experimentally computed multilevel asymptotic convergence factors (between brackets) for different values of parameter $\lambda$ and for increasing number of time steps, $M$, considering a fractional order $\delta=0.4$.

\begin{tabular}{ccccccc}
\hline $\log _{2} \lambda$ & $M=32$ & $M=64$ & $M=128$ & $M=256$ & $M=512$ & $M=1024$ \\
\hline-8 & 0.004 & 0.005 & 0.006 & 0.008 & 0.010 & 0.013 \\
& $(0.004)$ & $(0.005)$ & $(0.006)$ & $(0.009)$ & $(0.012)$ & $(0.014)$ \\
-6 & 0.016 & 0.018 & 0.023 & 0.028 & 0.036 & 0.045 \\
& $(0.017)$ & $(0.018)$ & $(0.027)$ & $(0.033)$ & $(0.041)$ & $(0.051)$ \\
-4 & 0.054 & 0.061 & 0.072 & 0.085 & 0.098 & 0.110 \\
& $(0.055)$ & $(0.065)$ & $(0.079)$ & $(0.088)$ & $(0.091)$ & $(0.112)$ \\
-2 & 0.116 & 0.120 & 0.125 & 0.128 & 0.132 & 0.134 \\
& $(0.122)$ & $(0.125)$ & $(0.135)$ & $(0.137)$ & $(0.137)$ & $(0.137)$ \\
0 & 0.114 & 0.117 & 0.119 & 0.120 & 0.121 & 0.121 \\
& $(0.120)$ & $(0.120)$ & $(0.120)$ & $(0.121)$ & $(0.122)$ & $(0.122)$ \\
2 & 0.054 & 0.058 & 0.060 & 0.062 & 0.062 & 0.063 \\
& $(0.057)$ & $(0.063)$ & $(0.063)$ & $(0.063)$ & $(0.063)$ & $(0.063)$ \\
4 & 0.016 & 0.017 & 0.019 & 0.019 & 0.019 & 0.020 \\
& $(0.020)$ & $(0.020)$ & $(0.020)$ & $(0.020)$ & $(0.020)$ & $(0.020)$ \\
6 & 0.004 & 0.004 & 0.005 & 0.005 & 0.005 & 0.005 \\
& $(0.005)$ & $(0.005)$ & $(0.005)$ & $(0.005)$ & $(0.005)$ & $(0.005)$ \\
8 & 0.001 & 0.001 & 0.001 & 0.001 & 0.001 & 0.001 \\
& $(0.001)$ & $(0.001)$ & $(0.001)$ & $(0.001)$ & $(0.001)$ & $(0.001)$ \\
\hline \multirow{2}{*}{6}
\end{tabular}

values of $M=2^{k}, k=5, \ldots, 10$, together with the experimentally computed asymptotic convergence factors obtained by using the multilevel $W$-cycle with one smoothing step. As expected, the predicted two-grid convergence factors provide a very accurate estimation of the real asymptotic convergence of the method. These results are shown for different values of parameter $\lambda$ and a fixed value of the fractional order $\delta=0.4$.

5. Fast implementation and computational cost. In Algorithm 1, we observe that the most time-consuming part of the WRMG method is the calculation of the defect and the smoothing step. The remaining components of the algorithm can be performed with a computational cost proportional to the number of unknowns. In the calculation of the residual, for each spatial grid point a matrix-vector multiplication 
$T_{M} x$ is required for some vector $x$, where $T_{M}$ is the low-triangular matrix

$$
T_{M}=\left(\begin{array}{cccc}
r_{1} & 0 & \cdots & 0 \\
r_{2} & r_{1} & \cdots & 0 \\
\vdots & \ddots & \ddots & \vdots \\
r_{M} & \cdots & r_{2} & r_{1}
\end{array}\right),
$$

with $r_{i}=\frac{\tau^{-\delta}}{\Gamma(2-\delta)}\left(d_{i}-d_{i-1}\right), i=1, \ldots, M$, assuming $d_{0}=0$.

Moreover, the smoothing part involves the solution of triangular linear systems. The matrix $\mathcal{A}_{h, \tau}$ of the discrete system to be solved can be written as $\mathcal{A}_{h, \tau}=T_{M} \otimes$ $I_{N}+A_{h}$, where $I_{N}$ denotes the identity matrix of order $N, A_{h}$ corresponds to the spatial discretization, $\otimes$ denotes the Kronecker product, and $T_{M}$ is the low-triangular matrix given in (27).

In a standard implementation, the method would have a computational cost of at least $O\left(N M^{2}\right)$ operations due to the matrix-vector multiplication $T_{M} x$ and the solution of the triangular systems in the smoothing part of the algorithm. However, due to the special structure of matrix $T_{M}$, the proposed multigrid waveform relaxation method can be implemented with a computational cost of $O(N M \log (M))$ operations, with a storage cost for the system matrix of $O(M)$. To see this, we discuss the following issues in the next subsections: a fast matrix-vector multiplication, a fast solution of the low-triangular systems, an efficient storage of matrix $\mathcal{A}_{h, \tau}$, and an estimation of the computational cost of the complete multigrid waveform relaxation method.

5.1. An $O(N M \log (M))$ calculation of the defect. To compute the residual in the WRMG method, a matrix-vector multiplication $\mathcal{A}_{h, \tau} u$ is required. The matrixvector multiplication corresponding to the spatial discretization can be calculated with a computational cost of $O(N M)$. Apart from this, for each spatial grid point we have to perform a matrix-vector multiplication $T_{M} x$ for some vector $x$. Notice that matrix $T_{M}$ is an $M \times M$ Toeplitz matrix, and therefore it can be embedded into a $2 M \times 2 M$ circulant matrix $C_{2 M}$ in the following way:

$$
C_{2 M}=\left(\begin{array}{cc}
T_{M} & R_{M} \\
R_{M} & T_{M}
\end{array}\right)
$$

where

$$
R_{M}=\left(\begin{array}{ccccc}
0 & r_{M} & r_{M-1} & \ldots & r_{2} \\
0 & 0 & r_{M} & \cdots & r_{3} \\
\vdots & \ddots & \ddots & \ddots & \vdots \\
0 & \ldots & 0 & \ldots & r_{M} \\
0 & 0 & 0 & \ldots & 0
\end{array}\right) .
$$

Taking into account that

$$
\left(\begin{array}{ll}
T_{M} & R_{M} \\
R_{M} & T_{M}
\end{array}\right)\left(\begin{array}{l}
x \\
0
\end{array}\right)=\left(\begin{array}{c}
T_{M} x \\
*
\end{array}\right)
$$

the matrix-vector multiplication is reduced to a circulant matrix-vector multiplication. It is known that a circulant matrix can be diagonalized by the Fourier matrix $F_{2 M}$ as $C_{2 M}=F_{2 M}^{*} D_{2 M} F_{2 M}$, where $D_{2 M}$ is a diagonal matrix whose diagonal elements are the eigenvalues of $C_{2 M}$. By taking the FFT of the first column of $C_{2 M}$, we can determine $D_{2 M}$ in $O(M \log (M))$ operations. Once $D_{2 M}$ is obtained, the multiplication $C_{2 M} v$ for some vector $v$ can be calculated by using a couple of FFTs with 
$O(M \log (M))$ complexity. As this is the computational cost for each spatial grid point, the product $\mathcal{A}_{h, \tau} u$ can be performed in $O(N M \log (M))$ operations.

5.2. An $O(N M \log (M))$ implementation of the smoothing procedure. Another one of the most consuming components of the WRMG method for solving the time-fractional diffusion equation is the relaxation step, since dense low-triangular systems must be solved. In the particular case of discrete problem (11), for each spatial grid point we need to solve a system of $M$ equations of the type $\left(T_{M}+2 / h^{2} I_{M}\right) x=b$ for some known vector $b$. Due to the Toeplitz structure of the matrix, the solution of the system can be obtained in $O(M \log (M))$ operations by using well-developed algorithms for the inversion of triangular Toeplitz matrices. With the inverse matrix obtained, which is again a Toeplitz matrix, the solution of the system is obtained by a matrix-vector multiplication with complexity of $O(M \log (M))$ operations by using the algorithm described in the previous subsection. Classical algorithms for the inversion of triangular Toeplitz matrices with complexity $O(M \log (M))$ include the Bini's algorithm [2], its revised version [25], and the divide and conquer method $[7,34]$. In our implementation, we have chosen the latter, which is briefly described to make this work more self-contained. A low-triangular Toeplitz matrix $T_{M}$, with $M=2^{p}, p>1$, can be partitioned as follows:

$$
T_{M}=\left(\begin{array}{ll}
T_{M / 2} & \\
P_{M / 2} & T_{M / 2}
\end{array}\right),
$$

where $T_{M / 2}$ and $P_{M / 2}$ are Toeplitz matrices of order $2^{p-1}$. Based on this partition, it is easy to see that the inverse of matrix $T_{M}$ can be written as

$$
T_{M}^{-1}=\left(\begin{array}{cc}
T_{M / 2}^{-1} & \\
-T_{M / 2}^{-1} P_{M / 2} T_{M / 2}^{-1} & T_{M / 2}^{-1}
\end{array}\right) .
$$

This expression gives us a recurrent method to calculate the inverse of matrix $T_{M}$. Since the inverse of this matrix is Toeplitz, it is enough to calculate its first column. Given a small number $p_{0}$, we compute the inverse of the submatrix $T_{2^{p_{0}}}$ by the forward substitution method, for instance. Then we subsequently apply the recurrent formula to compute the inverse of $T_{M}$ in $p-p_{0}$ steps. On each step the first column of the Toeplitz matrix $-T_{M / 2}^{-1} P_{M / 2} T_{M / 2}^{-1}$ is required, which can be calculated by FFTs. The total computational cost of the smoothing algorithm is therefore only $O(N M \log (M))$ at each iteration step. Moreover, since we need to solve several triangular systems with the same matrix but different right-hand sides, the first column of the inverse matrix can be computed a priori.

5.3. Storage cost and computational complexity of the multigrid waveform relaxation method. The nonlocal nature of the fractional derivatives results in a dense coefficient matrix yielding a bottleneck for the traditional numerical methods for fractional diffusion problems which require $O\left(M^{2}\right)$ units of storage. Due to the Toeplitz structure of matrix $T_{M}$ the memory requirement for the storage of the coefficient matrix can be significantly reduced to $O(M)$, since to perform all the calculations in our algorithm we only need to store its first column.

We consider a grid hierarchy $G_{0}, G_{1}, \ldots, G_{l}$, where $G_{k}:=G_{h_{k}, \tau}$ and $h_{0}>h_{1}>$ $\cdots>h_{l}$. It is well known that the computational work $\mathcal{W}_{l}$ per $V$-cycle on a grid $G_{l}$ is given by [45]

$$
\mathcal{W}_{l}=\sum_{k=1}^{l} \mathcal{W}_{k}^{k-1}+\mathcal{W}_{0}
$$


where $\mathcal{W}_{k}^{k-1}$ is the computational work of a two-grid cycle excluding the work needed to solve the defect equation on $G_{k}$, and $\mathcal{W}_{0}$ is the work needed to compute the exact solution on the coarsest grid $G_{0}$. In the computational work $\mathcal{W}_{k}^{k-1}$, included are the cost of a smoothing iteration, the calculation of the defect and its transfer to $G_{k-1}$, and the interpolation of the correction to $G_{k}$ and its addition to the previous approximation. From the previous subsections, we can estimate that the computational cost of a two-grid cycle is $\mathcal{W}_{k}^{k-1}=O\left(N_{k} M \log (M)\right)$ and on the coarsest grid $\mathcal{W}_{0}=O(M \log (M))$, where $N_{k}$ is the number of spatial grid points on the grid $G_{k}$ and $M$ is the number of time steps. Therefore, we can say that the computational cost of a $V$-cycle on level $l$ is roughly

$$
\mathcal{W}_{l}=\left(1+\frac{1}{2}+\frac{1}{2^{2}}+\cdots+\frac{1}{2^{l}}\right) O\left(N_{l} M \log (M)\right)=O\left(N_{l} M \log (M)\right) .
$$

Thus, since the $V$-cycle converges in a small number of iterations independent of the number of unknowns, the total computational cost for solving the time-fractional problem by the WRMG method is roughly $O\left(N_{l} M \log (M)\right)$.

6. Extension to two dimensions. This section is devoted to the extension of the presented methodology to problems with two spatial dimensions.

Model problem and discretization. We consider the two-dimensional timefractional diffusion equation as our model problem, that is,

$$
\begin{aligned}
D_{t}^{\delta} u-\Delta u & =f(x, y, t), \quad(x, y) \in \Omega \subset \mathbb{R}^{2}, t>0, \\
u(x, y, t) & =0, \quad(x, y) \in \partial \Omega, t>0, \\
u(x, y, 0) & =g(x, y), \quad(x, y) \in \bar{\Omega},
\end{aligned}
$$

where $\Delta$ denotes the two-dimensional Laplace operator, $\Omega$ is a square domain of length $L, \partial \Omega$ is its boundary, and $\bar{\Omega}=\Omega \cup \partial \Omega$. $D_{t}^{\delta}$ denotes again the Caputo fractional derivative,

$$
D_{t}^{\delta} u(x, y, t):=\left[\frac{1}{\Gamma(1-\delta)} \int_{0}^{t}(t-s)^{-\delta} \frac{\partial u(x, y, s)}{\partial s} d s\right], \quad(x, y) \in \Omega, t \geq 0 .
$$

Let us consider a uniform grid $G_{h, \tau}=G_{h} \times G_{\tau}$, with

$$
G_{h}=\left\{\left(x_{n}, y_{l}\right) \mid x_{n}=n h, y_{l}=l h, n, l=0,1, \ldots, N+1\right\},
$$

where $h=\frac{L}{N+1}$, and with $G_{\tau}$ given as in (7). The nodal approximation to the solution at each grid point $\left(x_{n}, y_{l}, t_{m}\right) \in G_{h, \tau}$ is denoted by $u_{n, l, m}$.

Standard central finite differences are used again to approximate the spatial derivatives, whereas the Caputo fractional derivative is discretized as

$$
D_{M}^{\delta} u_{n, l, m}:=\frac{\tau^{-\delta}}{\Gamma(2-\delta)}\left[d_{1} u_{n, l, m}-d_{m} u_{n, l, 0}+\sum_{k=1}^{m-1}\left(d_{k+1}-d_{k}\right) u_{n, l, m-k}\right],
$$

where coefficients $d_{k}$ are identically defined as in section 2 .

This results in the following discrete problem:

$$
\begin{aligned}
D_{M}^{\delta} u_{n, l, m}-\Delta_{h} u_{n, l, m} & =f\left(x_{n}, y_{l}, t_{m}\right), \quad 1 \leq n, l \leq N, 1 \leq m \leq M, \\
u_{n, l, m} & =0, \quad\left(x_{n}, y_{l}\right) \in \partial \Omega \cap G_{h, \tau}, 0<m \leq M, \\
u_{n, l, 0} & =g\left(x_{n}, y_{l}\right), \quad 0 \leq n, l \leq N+1,
\end{aligned}
$$

where

$$
\Delta_{h} u_{n, l, m}=\frac{u_{n+1, l, m}+u_{n, l+1, m}-4 u_{n, l, m}+u_{n-1, l, m}+u_{n, l-1, m}}{h^{2}} .
$$


Multigrid waveform relaxation in two dimensions. Regarding the solver for the considered two-dimensional time-fractional model problem (28)-(30), a redblack Gauss-Seidel waveform relaxation can be defined, after discretizing in space, as follows:

$$
\begin{aligned}
D_{t}^{\delta} u_{n, l}^{k}(t)+\frac{4}{h^{2}} u_{n, l}^{k}(t)=\frac{1}{h^{2}} & \left(u_{n-1, l}^{k-1}(t)+u_{n, l-1}^{k-1}(t)+u_{n+1, l}^{k-1}(t)+u_{n, l+1}^{k-1}(t)\right) \\
& +f_{n, l}(t) \text { if } n+l \text { is even, } \\
D_{t}^{\delta} u_{n, l}^{k}(t)-\frac{1}{h^{2}}\left(u_{n-1, l}^{k}(t)+\right. & \left.u_{n, l-1}^{k}(t)-4 u_{n, l}^{k}(t)+u_{n+1, l}^{k}(t)+u_{n, l+1}^{k}(t)\right) \\
= & f_{n, l}(t) \quad \text { if } n+l \text { is odd. }
\end{aligned}
$$

Thus, the fully discrete problem given in (34)-(36) can be solved by using an extension of the WRMG algorithm proposed in section 3. In this case, the method combines a two-dimensional coarsening strategy in the space variables and again a line-intime smoother based on the red-black Gauss-Seidel waveform relaxation; that is, the lines in time are visited following a red-black or chessboard manner. Regarding the intergrid transfer operators, the standard two-dimensional full-weighting restriction and bilinear interpolation are considered.

Semialgebraic mode analysis in two dimensions. The SAMA presented in section 4 can also be extended to study the convergence of the proposed multigrid waveform relaxation method. For this analysis, very little has to be changed from the theory developed in section 4 . The infinite grid $\mathcal{G}_{h}$ is defined as the extension of the spatial mesh given in (32), and then the grid functions defined on such a grid can again be expressed as formal linear combinations of the Fourier components, which in this case are given by the product of two complex exponential functions, i.e., $\varphi_{h}(\boldsymbol{\theta}, \mathbf{x})=e^{\imath \boldsymbol{\theta} \cdot \mathbf{x}}=e^{\imath \theta_{x} x} e^{\imath \theta_{y} y}$, where $\boldsymbol{\theta}=\left(\theta_{x}, \theta_{y}\right) \in \boldsymbol{\Theta}_{h}=(-\pi / h, \pi / h] \times(-\pi / h, \pi / h]$, and which form the new Fourier space. In the two-dimensional spatial case, it is well known that the Fourier space is decomposed in four-dimensional subspaces,

$$
\mathcal{F}^{4}(\boldsymbol{\theta})=\operatorname{span}\left\{\varphi_{h}\left(\boldsymbol{\theta}^{00}, \cdot\right), \varphi_{h}\left(\boldsymbol{\theta}^{11}, \cdot\right), \varphi_{h}\left(\boldsymbol{\theta}^{10}, \cdot\right), \varphi_{h}\left(\boldsymbol{\theta}^{01}, \cdot\right)\right\},
$$

generated by four Fourier modes associated with one low frequency, $\boldsymbol{\theta}=\boldsymbol{\theta}^{00} \in \Theta_{2 h}=$ $[-\pi / 2 h, \pi / 2 h)^{2}$, and three high frequencies, $\boldsymbol{\theta}^{11}, \boldsymbol{\theta}^{10}$, and $\boldsymbol{\theta}^{01}$, such that

$$
\boldsymbol{\theta}^{\alpha_{1}, \alpha_{2}}=\boldsymbol{\theta}^{00}-\left(\alpha_{1} \operatorname{sign}\left(\theta_{1}^{00}\right) \pi, \alpha_{2} \operatorname{sign}\left(\theta_{2}^{00}\right) \pi\right), \alpha_{1}, \alpha_{2} \in\{0,1\},
$$

which are coupled on the coarse grid by the aliasing effect.

Similarly as in the one-dimensional case, SAMA in two dimensions is based on a two-dimensional spatial LFA combined with an exact analysis in time. In this way, the resulting Fourier representations of the smoothing, coarse-grid, and two-grid operators are $4 M \times 4 M$ matrices.

Analysis results. Next, we present some results obtained by using the semialgebraic analysis. Similarly as we saw for the one-dimensional model problem, if we analyze the convergence of the method depending on parameter $\tau / h^{2}$, although the convergence rates are bounded by 0.25 for all cases, we do not obtain a $\delta$-independent convergence for a fixed value of $\tau / h^{2}$. This can be seen in Figure 4. However, we can show that the obtained multigrid convergence becomes robust for any value of $\delta$ with respect to parameter $\lambda=\tau^{\delta} \Gamma(2-\delta) / h^{2}$. This is shown in Figure 5 , where $M=32$ time levels have been considered, and the two-grid convergence factors predicted by 


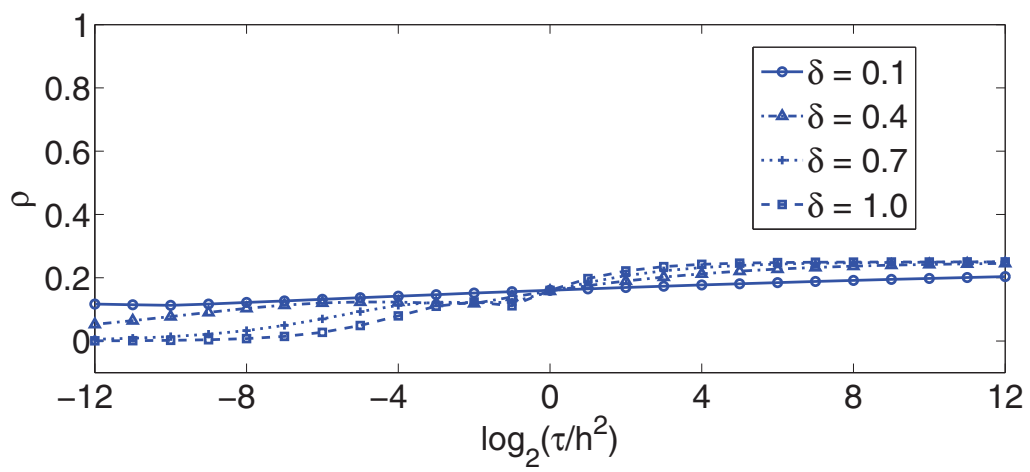

FIG. 4. Two-grid convergence factors predicted by the analysis for different values of parameter $\lambda=\tau / h^{2}$ and various fractional orders $\delta$.

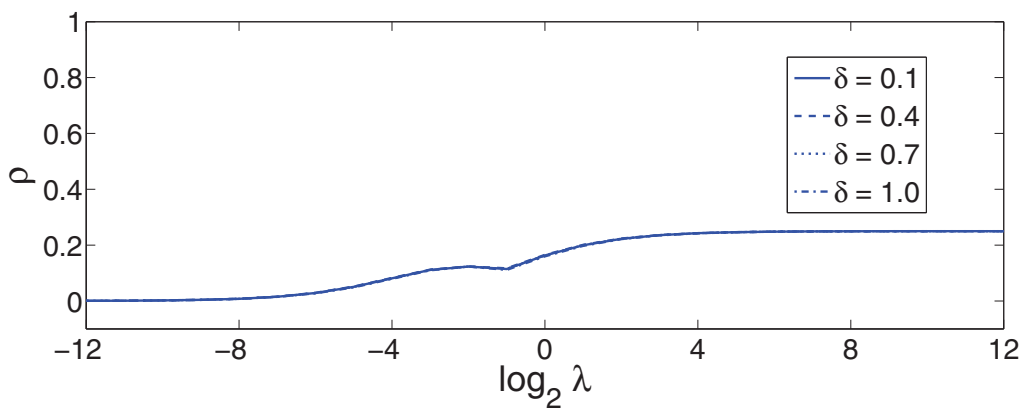

FIG. 5. Two-grid convergence factors predicted by the analysis for different values of parameter $\lambda=\tau^{\delta} \Gamma(2-\delta) / h^{2}$ and various fractional orders $\delta$.

the analysis for one smoothing step are shown for different values of parameter $\lambda$ and for different fractional orders $\delta$. Notice that the graphs corresponding to the different values of $\delta$ are almost indistinguishable, and for any value of $\lambda$ the multigrid convergence results are very satisfactory. These results can be confirmed with the asymptotic convergence factors experimentally computed. In particular, for $\delta=0.4$, we show this comparative in Figure 6, where the two-grid convergence factors predicted by SAMA are displayed together with the asymptotic convergence rates computed by using a $W(1,0)$-multigrid waveform relaxation algorithm on a fine grid of size $256 \times 256 \times 32$. Again, a random initial guess and a zero right-hand side are used to perform these calculations. Similar pictures can be obtained for other fractional orders $\delta$. We can observe a very accurate match between the analysis results and the rates experimentally obtained.

Remark. In Figures 3 (one-dimensional case) and 6 (two-dimensional case), a different behavior of the multigrid method is observed when $\lambda$ becomes big enough, that is, in the limit case of the steady problem. In that case, it is well known that the multigrid method based on a red-black smoother is an exact solver in the onedimensional case, whereas for a two-dimensional diffusion problem the convergence rate is about 0.25 for a $W$-cycle with one smoothing step (see [48]).

7. Numerical results. In this section, we consider three different numerical experiments to illustrate the efficiency of the proposed multigrid waveform relaxation 


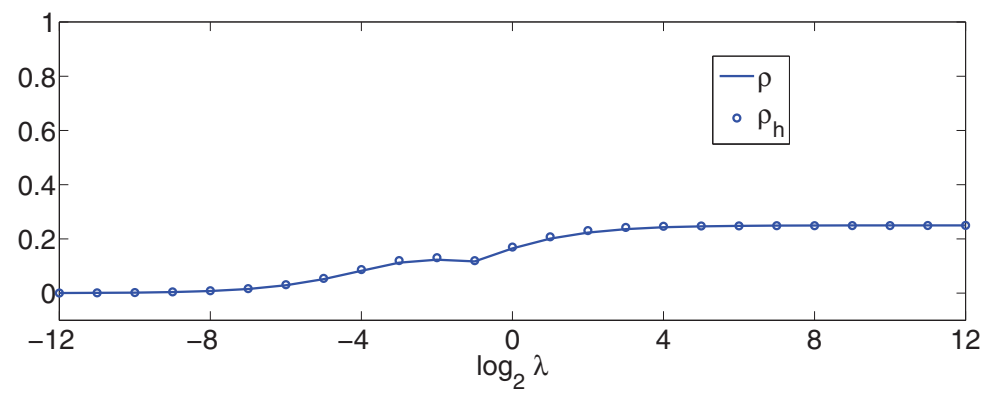

FIG. 6. Comparison between the two-grid convergence factors predicted by the analysis $(\rho)$ and the asymptotic convergence factor of a $W$-cycle experimentally computed $\left(\rho_{h}\right)$ for different values of parameter $\lambda=\tau^{\delta} \Gamma(2-\delta) / h^{2}$ and fractional order $\delta=0.4$.

method for solving the time-fractional heat equation. For all cases we perform $V$ cycles since they provide convergence rates similar to $W$-cycles, and therefore a more efficient multigrid method is obtained. We will start solving both one- and twodimensional linear problems, and finally we will solve a nonlinear one-dimensional problem. All numerical computations were carried out using MATLAB.

One-dimensional linear time-fractional heat equation. We show the efficient performance of the proposed multigrid waveform relaxation for a problem which considers reasonably general and realistic hypotheses on the behavior of the solution near the initial time. In particular, we consider a problem whose solution is smooth away from the initial time $(t=0)$, but it has a certain singular behavior at $t=0$ presenting a boundary layer. The theoretical convergence analysis of the considered finite difference discretization has been deeply studied in [46]. Here, we will show that the convergence of the WRMG is satisfactory for this representative model problem.

We consider problem (1)-(3) defined on a domain $[0, \pi] \times[0,1]$, with a zero righthand side $(f(x, t)=0)$ and an initial condition $g(x)=\sin x$. Then function $u(x, t)=$ $E_{\delta}\left(-t^{\delta}\right) \sin x$, where $E_{\delta}: \mathbb{R} \rightarrow \mathbb{R}$ is given by

$$
E_{\delta}(z):=\sum_{k=0}^{\infty} \frac{z^{k}}{\Gamma(\delta k+1)}
$$

satisfies our initial-boundary value problem $[28,46]$. In Figure 7 , we can observe the sharpness of the analytical solution near the initial time, where a boundary layer appears. In [46], it is proved rigorously that for "typical" solutions of (1)-(3) (no excessive smooth solutions) a rate of convergence of $\mathcal{O}\left(h^{2}+\tau^{\delta}\right)$ is obtained. This is shown in Figure 8 for four different values of $\delta$, where the maximum errors between the analytical and the numerical solutions are displayed for various numbers of time steps $M$ and assuming a sufficiently fine spatial grid. It can be seen that the slopes of the obtained graphs match with the expected convergence rates. For small values of $\delta$, a very fine temporal mesh would be required to attain the asymptotic rate of convergence, and this is the case of $\delta=0.1$ in the picture where a slow convergence of the rates to the expected asymptotic rate of 0.1 is observed.

Next, we show the independence of the convergence of the proposed WRMG method with respect to the discretization parameters. For this purpose, we consider different values of the fractional order $\delta$ and different grid sizes varying from $128 \times 128$ to $2048 \times 2048$, doubling the mesh size in both spatial and temporal dimensions. In 


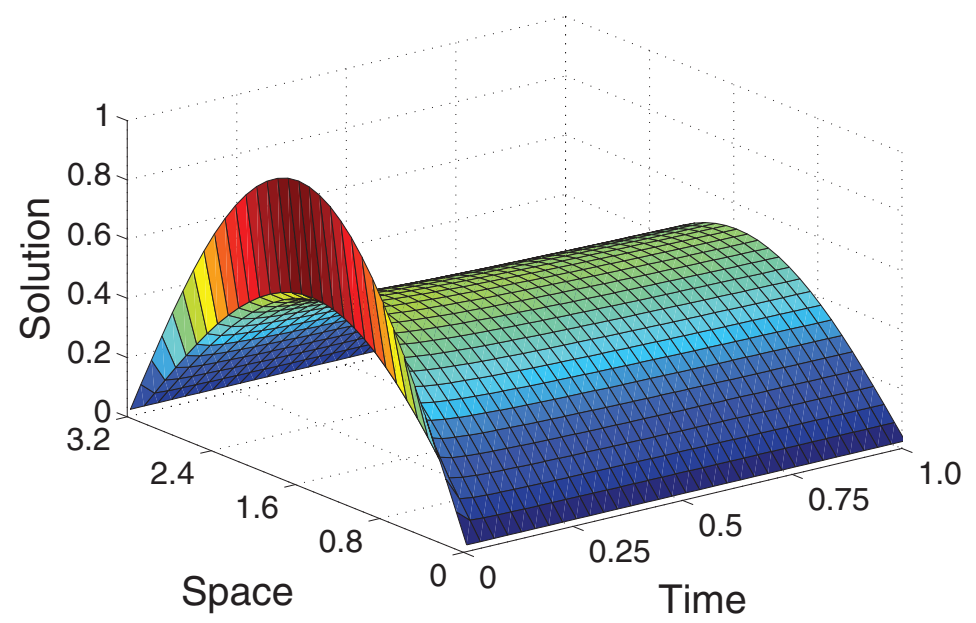

FIG. 7. Analytical solution $u(x, t)$ of the first test problem for fractional order $\delta=0.1$.

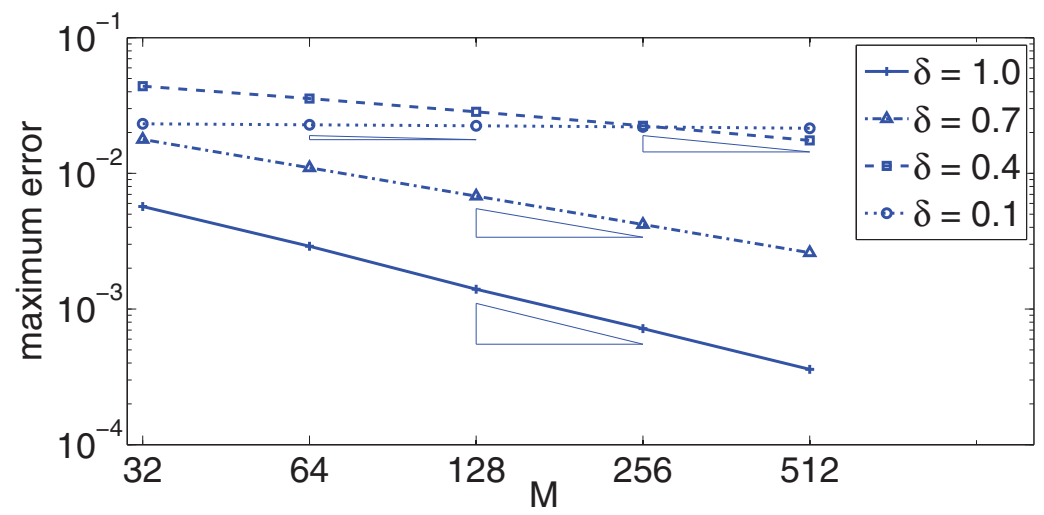

FIG. 8. Reduction of the maximum errors obtained for four different values of $\delta$ for the first test problem.

Table 2 we display the number of WRMG iterations necessary to reduce the initial residual by a factor of $10^{-10}$, together with the mean convergence factors and the corresponding CPU time, when considering a $V(0,1)$-cycle. We can observe that the performance of the $V$-cycle is also satisfactory for any value of $\delta$ and for increasing mesh sizes, as it was already seen for the $W$-cycle in the analysis results section. Moreover, we choose only one postsmoothing step since this approach provides much better convergence factors than a $V(1,0)$-cycle. Taking into account these considerations, we observe from Table 2 a robust convergence of the considered WRMG.

Two-dimensional linear time-fractional heat equation. The second numerical experiment deals with the solution of a two-dimensional linear time-fractional diffusion problem. We consider the following model problem defined on the spatial 
TABLE 2

Number of $V(0,1)-W R M G$ iterations necessary to reduce the initial residual by a factor of $10^{-10}$ for different fractional orders $\delta$ and for different grid sizes. The corresponding average convergence factors (in parentheses) and the CPU times in seconds are also included.

\begin{tabular}{cccccc}
\hline$\delta$ & $128 \times 128$ & $256 \times 256$ & $512 \times 512$ & $1024 \times 1024$ & $2048 \times 2048$ \\
\hline 0.1 & $8(0.03) 0.54 \mathrm{~s}$ & $8(0.03) 1 \mathrm{~s}$ & $8(0.03) 2.96 \mathrm{~s}$ & $8(0.03) 10.54 \mathrm{~s}$ & $7(0.03) 36.16 \mathrm{~s}$ \\
0.4 & $7(0.03) 0.49 \mathrm{~s}$ & $7(0.03) 0.91 \mathrm{~s}$ & $7(0.03) 2.60 \mathrm{~s}$ & $7(0.03) 9.31 \mathrm{~s}$ & $7(0.03) 36.34 \mathrm{~s}$ \\
0.7 & $7(0.04) 0.47 \mathrm{~s}$ & $7(0.04) 0.90 \mathrm{~s}$ & $7(0.04) 2.54 \mathrm{~s}$ & $7(0.04) 9.15 \mathrm{~s}$ & $7(0.04) 36.10 \mathrm{~s}$ \\
1.0 & $7(0.05) 0.46 \mathrm{~s}$ & $7(0.05) 0.88 \mathrm{~s}$ & $7(0.05) 2.54 \mathrm{~s}$ & $6(0.05) 7.96 \mathrm{~s}$ & $6(0.05) 30.69 \mathrm{~s}$ \\
\hline
\end{tabular}

domain $\Omega=(0,2) \times(0,2)$ :

$$
\begin{aligned}
D_{t}^{\delta} u-\Delta u & =f(x, y, t), \quad(x, y) \in \Omega, t>0 \\
u(x, y, t) & =0, \quad(x, y) \in \partial \Omega, t>0 \\
u(x, y, 0) & =0, \quad(x, y) \in \bar{\Omega}
\end{aligned}
$$

where

$$
f(x, y, t)=\left(\frac{2 t^{2-\delta}}{\Gamma(3-\delta)}+\left(1+\frac{\pi^{2}}{2}\right) t^{2}\right) \sin \frac{\pi x}{2} \sin \frac{\pi y}{2},
$$

in such a way that the analytic solution of the problem is

$$
u(x, y, t)=t^{2} \sin \frac{\pi x}{2} \sin \frac{\pi y}{2} .
$$

We consider the WRMG method described in section 6 by using a $V(1,1)$-cycle. This choice is based on the SAMA results presented in section 6. Due to the difference of the behavior of the method between the one- and two-dimensional problems, we have chosen two-smoothing steps to perform the calculations in this test case.

In Table 3 we display the number of WRMG iterations necessary to reduce the initial residual by a factor of $10^{-10}$ for different grid sizes varying from $32 \times 32 \times$ 32 to $256 \times 256 \times 256$ and for different values of the fractional order $\delta$. We can observe that the convergence of the proposed multigrid waveform relaxation is very robust with respect to the considered parameters. In the table, we also show the mean convergence factors and the corresponding CPU times. We can observe a very satisfactory convergence in all cases, making the WRMG method a good choice for an efficient solution of the time-fractional two-dimensional heat equation.

TABLE 3

Number of V(1,1)-WRMG iterations necessary to reduce the initial residual by a factor of $10^{-10}$, together with the corresponding average convergence factors (in parentheses) and the CPU times in seconds, for different fractional orders $\delta$ and for different grid sizes.

\begin{tabular}{ccccc}
\hline$\delta$ & $32 \times 32 \times 32$ & $64 \times 64 \times 64$ & $128 \times 128 \times 128$ & $256 \times 256 \times 256$ \\
\hline 0.1 & $12(0.10) 2.46 \mathrm{~s}$ & $12(0.10) 10.31 \mathrm{~s}$ & $12(0.11) 55.52 \mathrm{~s}$ & $12(0.11) 349.98 \mathrm{~s}$ \\
0.4 & $12(0.09) 2.51 \mathrm{~s}$ & $12(0.10) 10.45 \mathrm{~s}$ & $12(0.11) 55.86 \mathrm{~s}$ & $12(0.11) 348.29 \mathrm{~s}$ \\
0.7 & $11(0.09) 2.27 \mathrm{~s}$ & $12(0.10) 10.31 \mathrm{~s}$ & $12(0.11) 55.63 \mathrm{~s}$ & $12(0.11) 344.57 \mathrm{~s}$ \\
1.0 & $11(0.09) 2.29 \mathrm{~s}$ & $11(0.10) 9.68 \mathrm{~s}$ & $12(0.11) 55.73 \mathrm{~s}$ & $12(0.11) 346.44 \mathrm{~s}$ \\
\hline
\end{tabular}

One-dimensional nonlinear problem. The last numerical experiment is devoted to dealing with a nonlinear problem which appears in the modeling of anomalous 
diffusion in porous media $[14,42]$. We consider the following time-fractional PDE:

$$
D_{t}^{\delta} u=\frac{\partial}{\partial x}\left(D(u) \frac{\partial u}{\partial x}\right)+c \frac{\partial u}{\partial x}+f(x, t)
$$

where $D_{t}^{\delta}$ denotes again the Caputo fractional derivative operator with $0<\delta<1$, and $f(x, t)$ represents a source term. In this test problem we assume homogeneous Dirichlet boundary conditions and a zero initial condition.

Choosing $c=0$, model problem (42) has been used to describe the moisture distribution in construction materials [43], for example, whereas if the convective term is included, it is used to describe transport models for single-phase gas through tight rocks [30] or in groundwater hydrology [1]. For the discretization of problem (42), we consider again a uniform grid in space and time with step sizes $h$ and $\tau$, respectively. The fractional temporal derivative is discretized as previously by using the L1 scheme (see (10)). Regarding the spatial discretization, in an interior point $\left(x_{n}, t_{m}\right)$ the diffusion term is approximated by

$$
\frac{1}{h}\left[a_{n+1 / 2, m} \frac{u_{n+1, m}-u_{n, m}}{h}-a_{n-1 / 2, m} \frac{u_{n, m}-u_{n-1, m}}{h}\right],
$$

where $a_{n \pm 1 / 2, m}=\frac{1}{2}\left[D\left(u_{n \pm 1, m}\right)+D\left(u_{n, m}\right)\right]$, and for the convective term a standard upwind scheme is considered.

For the solution of the resulting discrete problem, we propose a nonlinear multigrid waveform relaxation method, derived from the well-known multigrid full-approximation scheme (FAS). This is the so-called waveform relaxation FAS method. This algorithm is easily derived from the standard FAS method [48] for solving elliptic equations. For a detailed description of the proposed algorithm we refer the reader to the book [49]. A nonlinear Gauss-Seidel waveform relaxation with a red-black ordering is considered, together with standard transfer-grid operators. Again, a $V(0,1)$-cycle is chosen to perform the calculations.

In Table 4, we show the convergence of the proposed algorithm for the case of $D(u)=1+u^{2}, c=1$, and $f(x, t)=1$ and for different values of the fractional order $\delta$. In particular, we display the number of iterations required to reduce the maximum initial residual by a factor of $10^{-10}$ for different grid sizes and the corresponding mean convergence factors (in parentheses). From the results in Table 4, we can conclude that the waveform relaxation FAS method shows behavior similar to that of the linear WRMG method for time-fractional diffusion problems.

TABLE 4

Number of $V(0,1)$-iterations of the waveform relaxation FAS method required to reduce the initial residual by a factor of $10^{-10}$ for different fractional orders $\delta$ and for different grid sizes, together with the corresponding mean convergence factors (in parentheses).

\begin{tabular}{ccccc}
\hline$\delta$ & $32 \times 32$ & $64 \times 64$ & $128 \times 128$ & $256 \times 256$ \\
\hline 0.1 & $11(0.09)$ & $11(0.10)$ & $11(0.10)$ & $12(0.10)$ \\
0.4 & $11(0.09)$ & $11(0.10)$ & $12(0.10)$ & $12(0.10)$ \\
0.7 & $11(0.09)$ & $11(0.10)$ & $12(0.10)$ & $12(0.10)$ \\
1.0 & $11(0.10)$ & $12(0.10)$ & $12(0.10)$ & $12(0.10)$ \\
\hline
\end{tabular}

8. Conclusions. A multigrid waveform relaxation method has been proposed for solving the time-fractional heat equation. The convergence of this method has 
been studied by a suitable semialgebraic mode analysis, which combines a classical exponential Fourier analysis in space with an algebraic computation in time. The results of this analysis show the efficiency and robustness of the proposed algorithm for the solution of the considered problem for different fractional orders. The proposed method has a computational cost of $O(N M \log (M))$ operations, where $M$ is the number of time steps and $N$ is the number of spatial grid points. Moreover, three numerical experiments confirm the good behavior of the WRMG method. In particular a linear one-dimensional representative problem, a linear two-dimensional model problem, and a nonlinear one-dimensional problem with applications in porous media are efficiently solved in this work.

Acknowledgment. The authors thank the referees for their valuable comments and suggestions, which helped to improve the paper.

\section{REFERENCES}

[1] B. Baeumer, S. Kurita, And M. Meerschaert, Inhomogeneous fractional diffusion equations, Fract. Calc. Appl. Anal., 8 (2005), pp. 371-386.

[2] D. BINI, Parallel solution of certain Toeplitz linear systems, SIAM J. Comput., 13 (1984), pp. 268-276, https://doi.org/10.1137/0213019.

[3] A. BRandt, Multi-level adaptive solutions to boundary-value problems, Math. Comp., 31 (1977), pp. 333-390.

[4] A. Brandt, Multigrid Solvers for Non-elliptic and Singular-Perturbation Steady-State Problems, tech. report, The Weizmann Institute of Science, Rehovot, Israel, 1981.

[5] A. BRAndt, Rigorous quantitative analysis of multigrid, I: Constant coefficients two-level cycle with $L_{2}$-norm, SIAM J. Numer. Anal., 31 (1994), pp. 1695-1730, https://doi.org/10.1137/ 0731087.

[6] H. Brunner, L. Ling, And M. Yamamoto, Numerical simulations of $2 D$ fractional subdiffusion problems, J. Comput. Phys., 229 (2010), pp. 6613-6622.

[7] D. Commenges and M. Monsion, Fast inversion of triangular Toeplitz matrices, IEEE Trans. Automat. Control, 29 (1984), pp. 250-251.

[8] J. H. Cushman and T. R. Ginn, Nonlocal dispersion in media with continuously evolving scales of heterogeneity, Transp. Porous Media, 13 (1993), pp. 123-138.

[9] P. J. Davis, Leonhard Euler's integral: A historical profile of the Gamma function, Amer. Math. Monthly, 66 (1959), pp. 849-869.

[10] K. Diethelm, The Analysis of Fractional Differential Equations. An Application-Oriented Exposition Using Differential Operators of Caputo Type, Lecture Notes in Math. 2004, Springer-Verlag, Berlin, 2010.

[11] R. Falgout, T. Manteuffel, B. Southworth, and J. Schroder, Parallel-in-Time for Moving Meshes, Report LLNL-TR-681918, Lawrence Livermore National Laboratory, 2016.

[12] S. Friedhoff and S. MacLachlan, A generalized predictive analysis tool for multigrid methods, Numer. Linear Algebra Appl., 22 (2015), pp. 618-647.

[13] F. J. Gaspar, C. Rodrigo, R. Ciegis, and A. Mirinavicius, Comparison of solvers for $2 D$ Schrodinger problems, Internat. J. Numer. Anal. Modeling, 11 (2014), pp. 131-147.

[14] E. Gerolymatou, I. Vardoulakis, and R. Hilfer, Modelling infiltration by means of a nonlinear fractional diffusion model, J. Phys. D Appl. Phys., 39 (2006), pp. 4104-4110.

[15] M. G. Hall and T. R. BARRICK, From diffusion-weighted MRI to anomalous diffusion imaging, Magnetic Resonance in Medicine, 59 (2008), pp. 447-455.

[16] R. D. Haynes and R. D. Russell, A Schwarz waveform moving mesh method, SIAM J. Sci. Comput., 29 (2007), pp. 656-673, https://doi.org/10.1137/050631549.

[17] R. Hilfer, Applications of Fractional Calculus in Physics, World Scientific, Singapore, 2000.

[18] G. Horton, S. Vandewalle, And P. Worley, An algorithm with polylog parallel complexity for solving parabolic partial differential equations, SIAM J. Sci. Comput., 16 (1995), pp. 531-541, https://doi.org/10.1137/0916034.

[19] Y.-L. JiANG AND X.-L. Ding, Waveform relaxation methods for fractional differential equations with the Caputo derivatives, J. Comput. Appl. Math., 238 (2013), pp. 51-67.

[20] B. Jin, R. LAZARov, J. PASCIAK, AND Z. Zhou, Error analysis of semidiscrete finite element methods for inhomogeneous time-fractional diffusion, IMA J. Numer. Anal., 35 (2015), pp. 561-582. 
[21] B. Jin, R. Lazarov, And Z. Zhou, Two fully discrete schemes for fractional diffusion and diffusion-wave equations with nonsmooth data, SIAM J. Sci. Comput., 38 (2016), pp. A146A170, https://doi.org/10.1137/140979563.

[22] R. Ke, M. K. NG, AND H.-W. Sun, A fast direct method for block triangular Toeplitz-like with tri-diagonal block systems from time-fractional partial differential equations, J. Comput. Phys., 303 (2015), pp. 203-211.

[23] X. Lei Lin, X. Lu, M. K. NG, And H.-W. Sun, A fast accurate approximation method with multigrid solver for two-dimensional fractional sub-diffusion equation, J. Comput. Phys., 323 (2016), pp. 204-218.

[24] X. Li AND C. XU, Existence and uniqueness of the weak solution of the space-time fractional diffusion equation and a spectral method approximation, Commun. Comput. Phys., 8 (2010), pp. 1016-1051.

[25] F. Lin, W. Ching, And M. NG, Fast inversion of triangular Toeplitz matrices, Theoret. Comput. Sci., 315 (2004), pp. 511-523.

[26] Y. Lin AND C. XU, Finite difference/spectral approximations for the time-fractional diffusion equation, J. Comput. Phys., 225 (2007), pp. 1533-1552.

[27] C. Lubich and A. Ostermann, Multigrid dynamic iteration for parabolic equations, BIT, 27 (1987), pp. 216-234.

[28] Y. LuChKo, Initial-boundary-value problems for the one-dimensional time-fractional diffusion equation, Fract. Calc. Appl. Anal., 15 (2012), pp. 141-160.

[29] J. T. Machado, V. Kiryakova, and F. Mainardi, Recent history of fractional calculus, Commun. Nonlinear Sci. Numer. Simul., 16 (2011), pp. 1140-1153.

[30] N. A. Malik, I. Ali, B. Chanane, and R. A. Ghanam, Time fractional transport model for flow through tight porous media, in Proceedings of the Sixth International Conference on Porous Media and Its Applications in Science, Engineering and Industry, ECI Symposium Series, 2016.

[31] M. M. Meerschaert and C. Tadjeran, Finite difference approximations for two-sided spacefractional partial differential equations, Appl. Numer. Math., 56 (2006), pp. 80-90.

[32] R. Metzler and J. Klafter, The random walk's guide to anomalous diffusion: A fractional dynamics approach, Phys. Rep., 339 (2000), pp. 1-77.

[33] U. MiekKala And O. Nevanlinna, Convergence of dynamic iteration methods for initial value problems, SIAM J. Sci. Statist. Comput., 8 (1987), pp. 459-482, https://doi.org/10.1137/ 0908046.

[34] M. MoRf, Doubling algorithms for Toeplitz and related equations, in Proceedings of the IEEE International Conference on Acoustics, Speech and Signal Processing (ICASSP), IEEE, 1980, pp. 954-959.

[35] K. Mustapha AND W. MCLean, Uniform convergence for a discontinuous Galerkin, timestepping method applied to a fractional diffusion equation, IMA J. Numer. Anal., 32 (2012), pp. 906-925.

[36] Y.-N. Zhang And Z.-Z. Sun, Error analysis of a compact ADI scheme for the $2 D$ fractional subdiffusion equation, J. Sci. Comput., 59 (2014), pp. 104-128.

[37] K. Oldham and J. Spanier, The Fractional Calculus: Theory and Applications of Differentiation and Integration to Arbitrary Order, Dover Books on Mathematics, Dover, 2006.

[38] C. Oosterlee, F. Gaspar, T. Washio, and R. Wienands, Multigrid line smoothers for higher order upwind discretizations of convection-dominated problems, J. Comput. Phys., 139 (1998), pp. 274-307.

[39] C. Oosterlee And P. Wesseling, Multigrid schemes for time-dependent incompressible Navier-Stokes equations, IMPACT Comput. Sci. Engrg., 5 (1993), pp. 153-175.

[40] J. Pan, R. Ke, M. K. NG, And H.-W. Sun, Preconditioned techniques for diagonal-timesToeplitz matrices in fractional diffusion equations, SIAM J. Sci. Comput., 36 (2014), pp. A2698-A2719, https://doi.org/10.1137/130931795.

[41] H.-K. PAng AND H.-W. Sun, Multigrid method for fractional diffusion equations, J. Comput. Phys., 231 (2012), pp. 693-703.

[42] Ł. PŁociniczak, Approximation of the Erdélyi-Kober operator with application to the timefractional porous medium equation, SIAM J. Appl. Math., 74 (2014), pp. 1219-1237, http://dx.doi.org/10.1137/130942450.

[43] Ł. PŁociniczak, Analytical studies of a time-fractional porous medium equation. Derivation, approximation and applications, Commun. Nonlinear Sci. Numer. Simul., 24 (2015), pp. $169-183$.

[44] S. D. Purohit, Solutions of fractional partial differential equations of quantum mechanics, Adv. Appl. Math. Mech., 5 (2013), pp. 639-651.

[45] K. Stüben and U. Trottenberg, Multigrid methods: Fundamental algorithms, model problem 
analysis and applications, in Multigrid Methods, Lecture Notes in Math. 960, W. Hackbusch and U. Trottenberg, eds., Springer, Berlin, Heidelberg, 1982, pp. 1-176.

[46] M. Stynes, E. O'Riordan, And J. L. Gracia, Error analysis of a finite difference method on graded meshes for a time-fractional diffusion equation, SIAM J. Numer. Anal., 55 (2017), pp. 1057-1079, https://doi.org/10.1137/16M1082329.

[47] J. Tenreiro Machado, A. M. Galhano, and J. J. Trujillo, Science metrics on fractional calculus development since 1966, Fract. Calc. Appl. Anal., 16 (2013), pp. 479-500.

[48] U. Trottenberg, C. W. Oosterlee, and A. Schüller, Multigrid, Academic Press, New York, 2001.

[49] S. Vandewalle, Parallel Multigrid Waveform Relaxation for Parabolic Problems, B.G. Teubner, Stuttgart, 1993.

[50] S. VANDEWALle AND G. Horton, Fourier mode analysis of the multigrid waveform relaxation and time-parallel multigrid methods, Computing, 54 (1995), pp. 317-330.

[51] S. Vandewalle and R. Piessens, Numerical experiments with nonlinear multigrid waveform relaxation on a parallel processor, Appl. Numer. Math., 8 (1991), pp. 149-161.

[52] S. VANdewalle And R. Piessens, Efficient parallel algorithms for solving initial-boundary value and time-periodic parabolic partial differential equations, SIAM J. Sci. Statist. Comput., 13 (1992), pp. 1330-1346.

[53] S. Vandewalle and R. Piessens, On dynamic iteration methods for solving time-periodic differential equations, SIAM J. Numer. Anal., 30 (1993), pp. 286-303, https://doi.org/10. $1137 / 0730014$.

[54] H. Wang ANd T. S. Basu, A fast finite difference method for two-dimensional space-fractional diffusion equations, SIAM J. Sci. Comput., 34 (2012), pp. A2444-A2458, https://doi.org/ $10.1137 / 12086491 \mathrm{X}$.

[55] H. Wang, K. Wang, And T. Sircar, A direct $O\left(N \log ^{2} N\right)$ finite difference method for fractional diffusion equations, J. Comput. Phys., 229 (2010), pp. 8095-8104.

[56] P. Wesseling, An Introduction to Multigrid Methods, John Wiley, Chichester, UK, 1992.

[57] R. Wienands and W. Joppich, Practical Fourier Analysis for Multigrid Methods, Chapman \& Hall/CRC Press, Boca Raton, FL, 2005.

[58] X. Zhao, X. Hu, W. Cai, and G. Karniadakis, Adaptive finite element method for fractional differential equations using hierarchical matrices, Comput. Methods Appl. Mech. Engrg., submitted. 